\title{
Stabilized continuous and discontinuous Galerkin techniques for Darcy flow
}

\author{
Santiago Badia*, Ramon Codina \\ International Center for Numerical Methods in Engineering (CIMNE), Universitat Politècnica de Catalunya, Jordi Girona 1-3, Edifici C1, 08034 Barcelona, Spain
}

\section{A R T I C L E I N F O}

\section{Article history:}

Received 16 September 2009

Received in revised form 21 January 2010

Accepted 22 January 2010

Available online 9 March 2010

\section{Keywords:}

Darcy's problem

Stabilized finite element methods

Characteristic length scale

Orthogonal subgrid scales

\begin{abstract}
A B S T R A C T
We design stabilized methods based on the variational multiscale decomposition of Darcy's problem. A model for the subscales is designed by using a heuristic Fourier analysis. This model involves a characteristic length scale, that can go from the element size to the diameter of the domain, leading to stabilized methods with different stability and convergence properties. These stabilized methods mimic different possible functional settings of the continuous problem. The optimal method depends on the velocity and pressure approximation order. They also involve a subgrid projector that can be either the identity (when applied to finite element residuals) or can have an image orthogonal to the finite element space. In particular, we have designed a new stabilized method that allows the use of piecewise constant pressures. We consider a general setting in which velocity and pressure can be approximated by either continuous or discontinuous approximations. All these methods have been analyzed, proving stability and convergence results. In some cases, duality arguments have been used to obtain error bounds in the $L^{2}$-norm.
\end{abstract}

(c) 2010 Elsevier B.V. All rights reserved.

\section{Introduction}

Darcy's problem governs the flow of an incompressible fluid through a porous medium. It is composed by the Darcy law that relates the fluid velocity (the flux) and the pressure gradient and the mass conservation equation. In flow in porous media, a proper functional setting for this problem is to consider the flux in $H(\operatorname{div}, \Omega$ ) and the pressure in $L^{2}(\Omega)$. This yields a saddle-point problem that is well posed due to inf-sup conditions known to hold at the continuous level, and that allow one to obtain stability estimates for the pressure and the velocity divergence.

The Galerkin approximation of this indefinite system is a difficult task, because the continuous inf-sup conditions are not naturally inherited by most finite element (FE) velocity-pressure spaces. We can avoid these problems by invoking the Darcy law in the mass conservation equation, getting a pressure Poisson problem; this is an elliptic problem that can be easily approximated by the Galerkin technique and Lagrangian elements. The fluxes can be obtained as a postprocess by using a $L^{2}$-projection. This approach is computationally appealing because pressure and velocity computations are decoupled and the implementation is easy. Unfortunately, this approach has two drawbacks: the loss of accuracy for the velocity and the very weak enforcement of the mass conservation equation. Improved post-processing techniques that reduce these problems can be found e.g. in Refs. [15,17]. This approach has been restricted to continuous ( $H^{1}$-conforming) pressure FE spaces. However, the

\footnotetext{
* Corresponding author.

E-mail addresses: sbadia@cimne.upc.edu (S. Badia),ramon.codina@upc.edu (R. Codina).
}

continuous pressure admits discontinuities, e.g. in regions with jumps of the physical properties (conductivity), and this approach leads to poor accuracy in the vicinity of these regions.

The indefinite problem can be approximated by the Galerkin technique and mixed FE formulations (see Ref. [5]) that satisfy the inf-sup conditions required for the well-posedness of the discrete problem. As an example, the combination of the Raviart-Thomas FE velocity space introduced in Ref. [25] with piecewise constant or linear pressures leads to stable approximations. The Raviart-Thomas FE space is $H(\operatorname{div}, \Omega)$-conforming; it is composed by vector functions with continuous normal traces and discontinuous tangential traces on the element boundaries, even though discontinuous Galerkin RaviartThomas FE methods have recently been proposed in Ref. [8]. The element unknowns are the normal fluxes on the faces, but all components are needed inside every element domain. This makes the implementation involved, especially for three dimensional problems. On the other hand, this FE space experiments a loss of accuracy in some meshes (see Ref. [2]). Finally, when dealing with a coupled Stokes-Darcy problem it is hard to find mixed FE methods that are stable for both the Stokes and the Darcy problems (see Refs. [1,22]). The FE spaces that satisfy these conditions are expensive and restricted to particular typologies of meshes that complicate their use in real applications. For the same reasons, they are not appealing when solving the Biot system that couples in a particular way the elastic problem and the Darcy problem (possibly coupled with the Navier-Stokes equations too).

A third alternative is to resort to stabilization techniques that perturb the indefinite problem in such a way that the FE approximation can violate the inf-sup condition in the functional setting of the continuous problem. Stabilization techniques for the Darcy problem 
have been designed in Ref. [23]. Therein, the stabilized problem mimics the mixed Laplacian functional setting (the pressure belongs to $H^{1}(\Omega)$ and the velocity belongs to $L^{2}(\Omega)$ ) and leads to the same order of convergence that is attained when using the pressure Poisson problem plus postprocessing. This method has been extended to discontinuous FE spaces for velocities and pressures in Refs. [6,20]. The stabilization term is the inner product of the residual times the adjoint of the Darcy differential operator applied to the test function. Correa and Loula have considered an interesting stabilized conforming finite element formulation in Ref. [16] that gives very strong stability bounds; both velocity and pressure are in $H^{1}(\Omega)$. The authors use the continuous embedding of $H^{1}(\Omega)$ in $H($ curl, $\Omega) \cap H(\operatorname{div}, \Omega)$. However, no convergence is attained for the natural norm and only $L^{2}$ norms of the errors can be bounded using elliptic regularity properties. So, the error estimates do not apply for non-convex domains. On the other hand, strong continuity assumptions over the permeability have to be assumed, which are not true for heterogeneous media.

In this work, we motivate stabilized methods based on the variational multiscale (VMS) decomposition of the Darcy problem which is in fact an adjoint formulation (see Refs. [19,24]). A matrix of algorithmic stabilization parameters appears, which we design using a heuristic Fourier analysis. The definition of this matrix involves a characteristic length scale. The choice of this characteristic length, which can be either the element size or the diameter of the domain, leads to stabilized methods with different stability and convergence properties. In this frame, we get numerical methods that mimic the typical setting in Darcy's flow (the velocity belongs to $H($ div, $\Omega$ ) and

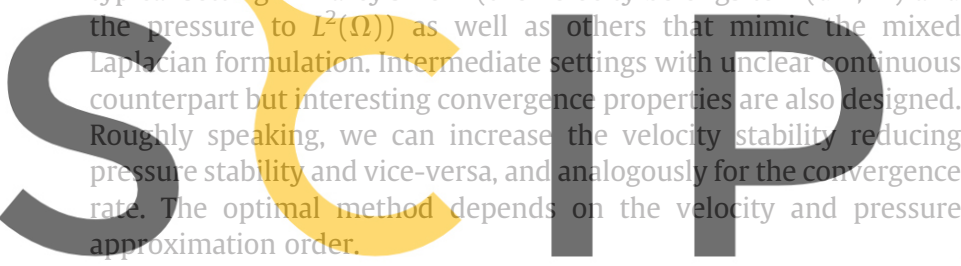

The methods motivated by VMS also involve a subgrid projection of the residual of the finite element solution. If the subgrid projection

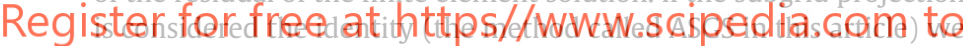
recover, up to the definition of the stabilization parameters, the methods discussed in Refs. $[20,23,24]$. We will also consider the case in which the subgrid projection is orthogonal to the finite element space (the method termed OSS below), as suggested in Ref. [9]. We thus motivate in a unified way a wide set of stabilized methods that can keep symmetry and mimic the different functional settings of the continuous problem (as well as other methods). In particular, we suggest a new stabilized method that allows the use of piecewise constant pressure - as far as we know, the first of this kind.

We have considered a general setting in which velocity and pressure can be approximated by using either continuous or discontinuous approximations. All these methods have been analyzed, proving stability and convergence results. In some cases, AubinNitsche-type duality arguments have been used to obtain error bounds in the $L^{2}$-norm. We have previously suggested a unified stabilization of the coupled Stokes-Darcy problem and performed the numerical analysis in Ref. [4] using these ideas.

Let us give the outline of the paper. In Section 2 we introduce the continuous problem and analyze its stability. Section 3 introduces a (non-conforming) discontinuous Galerkin (dG) approximation of the problem. We motivate the stabilization methods in the VMS framework and suggest and expression for the stabilization parameters and subgrid projector in Section 4. Section 5 is devoted to the stability and convergence analysis of these stabilized FE approximations. Improved error estimates obtained by duality arguments are presented in Section 6. We draw some recommendations about the method to use in Section 7, depending on the order of approximation of velocities and pressures. Numerical tests that show experimental convergence rates can be found in Section 8. We close the paper with some conclusions.

\section{Continuous problem}

\subsection{Problem statement}

Let $\Omega \subset \mathbb{R}^{d}, d=2,3$, be a polyhedral domain with Lipschitz boundary, denoted by $\Gamma$, where we consider the Darcy problem, which consists in finding a velocity $\boldsymbol{u}: \Omega \rightarrow \mathbb{R}^{d}$ and a pressure $p: \Omega \rightarrow \mathbb{R}$ (defined up to a constant) such that

$\sigma \boldsymbol{u}+\nabla p=\boldsymbol{f}$,

$\nabla \cdot \boldsymbol{u}=g$

where $\boldsymbol{f}$ and $g$ are given functions and the physical parameter $\sigma$ is the inverse of the permeability. ${ }^{1}$ As boundary conditions we will consider $n \cdot u=\psi$ on $\Gamma, n$ being the unit exterior normal. Obviously, the compatibility condition $\int \Gamma \psi d \Gamma=\int \Omega g d \Omega$ must be satisfied. The body force $f$ is usually zero for flow in porous media. However, we will keep $\boldsymbol{f}$ because a non-zero $\boldsymbol{f}$ is needed for some interesting applications governed by system (1), like in magnetohydrodynamics, where the current density is governed by Ohm's law and the conservation of charge.

Let us introduce some standard notation. The space of functions whose $p$ power $(1 \leq p<\infty)$ is integrable in a domain $\omega$ is denoted by $L^{p}(\omega), L^{\infty}(\omega)$ being the space of bounded functions in $\omega$ (in the

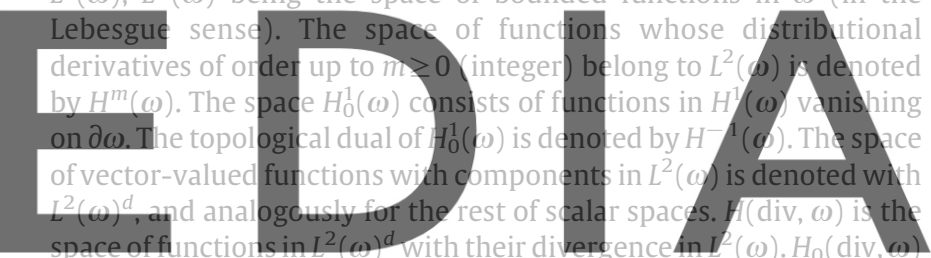

is the space of vector fields in $H($ div, $\omega)$ with zero normal trace on $\partial \omega$. download the version without the waternark of $H^{1 / 2}(\beta)$ is the space of fluxes denoted by $H^{-1 / 2}(\beta)$.

The Darcy problem can be thought in two different ways:

1. The dual mixed formulation, the typical setting for flow in porous media:

$$
\begin{aligned}
\boldsymbol{u} \in H(\operatorname{div}, \Omega), & p \in L^{2}(\Omega) / \mathbb{R}, \\
\boldsymbol{f} \in H(\operatorname{div}, \Omega)^{\prime}, & g \in L^{2}(\Omega), \quad \psi \in L^{2}(\Gamma)
\end{aligned}
$$

with the essential boundary condition $\boldsymbol{n} \cdot \boldsymbol{u}=\psi$.

2. The primal mixed formulation, which consists in a mixed formulation of the Poisson problem. In this case, the functional setting is:

$$
\begin{array}{ll}
\boldsymbol{u} \in L^{2}(\Omega)^{d}, & p \in H^{1}(\Omega) / \mathbb{R}, \\
\boldsymbol{f} \in L^{2}(\Omega)^{d}, & g \in H^{-1}(\Omega), \quad \psi \in H^{-1 / 2}(\Gamma) .
\end{array}
$$

Note that for an arbitrary function $\boldsymbol{v} \in L^{2}(\Omega)^{d}$, the normal trace of $\boldsymbol{v}$ is not defined and cannot be enforced. The boundary condition $\boldsymbol{n} \cdot \boldsymbol{u}=\psi$ (which is essential in the previous setting) is natural and holds in $H^{-1 / 2}(\Gamma)$. In this case, (essential) pressure boundary conditions can be imposed too, since the pressure trace belongs to $H^{1 / 2}(\Gamma)$.

\footnotetext{
1 The permeability is in general a tensor. For the sake of simplicity in the following exposition, let us consider the homogeneous case, in which the permeability can be considered to be a scalar. In the most general case, the following exposition is straightforward after minor modifications.
} 
In fact, whichever the situation is, it will be determined by the data. In the next subsection we will obtain an inf-sup condition that can be trivially translated into velocity-pressure stability if the data are regular enough. For the sake of clarity we have considered $\sigma$ to be a positive constant, but all the results obtained in this work apply for the general case in which $\sigma \in L^{\infty}(\Omega)$ and $\sigma_{+} \geq \sigma(\boldsymbol{x}) \geq \sigma_{-}>0$ for all $\boldsymbol{x} \in \Omega$ (up to sets of zero measure), where $\sigma_{+}$and $\sigma_{-}$are constants.

Let us denote by $\left\langle f_{1}, f_{2}\right\rangle$ the integral of two (generalized) functions $f_{1}$ and $f_{2}$ (either scalar or vector-valued) in $\Omega$. The regularity of both is such that the integral is well defined. For example, if $f_{1} \in H_{0}^{1}(\Omega)$ we may take $f_{2} \in H^{-1}(\Omega)$. When both $f_{1}, f_{2} \in L^{2}(\Omega)$ we will write their $L^{2}(\Omega)$ inner product as $\left\langle f_{1}, f_{2}\right\rangle \equiv\left(f_{1}, f_{2}\right)$. The associated norm will be denoted by $\left\|f_{1}\right\|_{L^{2}(\Omega)} \equiv\left\|f_{1}\right\|$. For two functions $\psi_{1}$ and $\psi_{2}$ defined on the boundary, we denote their integral over $\Gamma$ by $\left\langle\psi_{1}, \psi_{2}\right\rangle_{\text {}}$, assuming it makes sense. For example, for $\psi_{1}$ in $H^{\frac{1}{2}}(\Gamma), \psi_{2}$ must belong to $H^{-\frac{1}{2}}(\Gamma)$.

In the situation (2), the variational formulation of the problem consists in finding a velocity-pressure pair $[\boldsymbol{u}, p] \in H(\operatorname{div}, \Omega) \times L^{2}(\Omega) / \mathbb{R}$, with $n \cdot \boldsymbol{u}=\psi$ on $\Gamma$, such that

\section{$B_{c}([\boldsymbol{u}, p],[\boldsymbol{v}, q])=L_{c}([\boldsymbol{v}, q])$ \\ for all the $[\boldsymbol{v}, q]$ in the test space $H_{0}(\operatorname{div}, \Omega) \times L^{2}(\Omega)$, where the bilinear form $B_{c}$ and the linear form $L_{c}$ are defined by \\ $B_{c}([\boldsymbol{u}, p],[\boldsymbol{v}, q])=\sigma(\boldsymbol{u}, \boldsymbol{v})-(p, \nabla \cdot \boldsymbol{v})+(q, \nabla \cdot \boldsymbol{u})$

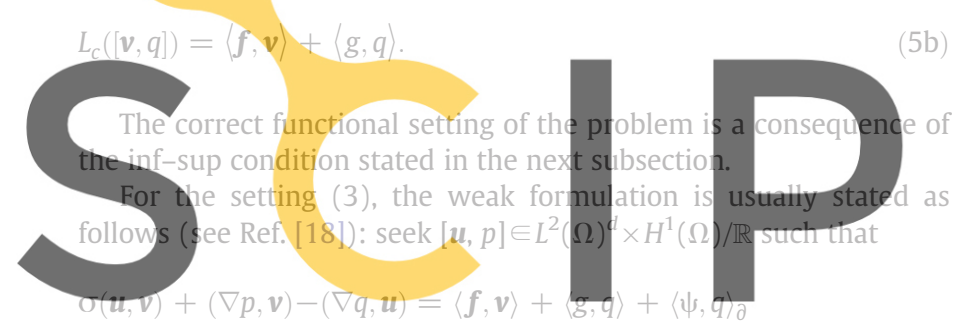

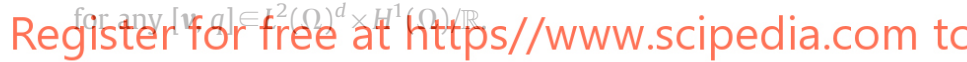

\subsection{A priori stability bounds}

A key ingredient in the following discussion is the introduction of a characteristic length scale of the problem, that we denote by $L_{0}$, which may be taken as the diameter of the computational domain $\Omega$. Whereas for the Stokes problem its introduction is unnecessary, it will play a key role in the Darcy problem. The ultimate reason to explain this fact is that in the Stokes case the seminorm $\|\nabla \boldsymbol{u}\|$ controls the whole norm in $H_{0}^{1}(\Omega)^{d}$ because of the Poincaré-Friedrichs inequality, and thus a stability estimate in this seminorm suffices; an analogous situation occurs for the elastic problem and Korn's inequality (see Ref. [7]). However, for the Darcy problem we need to control both $\boldsymbol{u}$ and $\nabla \cdot \boldsymbol{u}$ to obtain stability in $H(\operatorname{div}, \Omega)$, and the only way to incorporate both norms in a dimensionally correct one is through the introduction of a length scale. Thus, we introduce the following norm:

$\|\boldsymbol{v}\|_{H(\operatorname{div}, \Omega)}=\|\boldsymbol{v}\|+L_{0}\|\nabla \cdot \boldsymbol{v}\|$

While this discussion might seem unnecessary to obtain theoretical stability estimates (and thus to determine the functional framework of the problem), it will lead to very important consequences in the discrete finite element problem.

The correct functional setting of the problem (Eqs. (4)-(5)) is a consequence of the inf-sup condition

$\inf _{q \in L^{2}(\Omega)} \sup _{\boldsymbol{v} \in H(\operatorname{div}, \Omega)} \frac{(q, \nabla \cdot \boldsymbol{v})}{\|q\|\left(\|\boldsymbol{v}\|+L_{0}\|\nabla \cdot \boldsymbol{v}\|\right)} \geq \beta>0$ which is true due to the surjectivity of the divergence operator from $H(\operatorname{div}, \Omega)$ onto $L^{2}(\Omega)$ (see e.g. Ref. [18]).

Let now $V$ (the velocity space) be the closure of $C^{\infty}(\Omega)^{d}$ with respect to the norm $\sqrt{\sigma}\|\boldsymbol{v}\|+\sqrt{\sigma} L_{0}\|\nabla \cdot \boldsymbol{v}\|$ and $Q$ the closure of $C^{\infty}(\Omega) / \mathbb{R}$ with respect to $\left(\sqrt{\sigma} L_{0}\right)^{-1}\|q\|$. The pair $V \times Q$ reduces to $H(\operatorname{div}, \Omega) \times L^{2}(\Omega) / \mathbb{R}$. On this space we define

$\|[\boldsymbol{v}, q]\|_{c}^{2}:=\sigma\|\boldsymbol{v}\|^{2}+\sigma L_{0}^{2}\|\nabla \cdot \boldsymbol{v}\|^{2}+\frac{1}{\sigma L_{0}^{2}}\|q\|^{2}$.

We will denote by $V_{\psi}$ the subspace of $V$ of functions $\boldsymbol{v} \in V$ such that $\boldsymbol{n} \cdot \boldsymbol{v}=\psi$, and $V_{0}$ the subspace of functions such that $\boldsymbol{n} \cdot \boldsymbol{v}=0$. For the sake of simplicity, $\psi=0$ is considered in the following theorem, although non-homogeneous conditions will be taken into account at the discrete level.

In what follows, $C$ denotes a positive constant, in our case independent of $\sigma$ and $L_{0}$. When dealing with the finite element problem, $C$ will be independent also of the mesh size $h$. The value of $C$ may be different at different occurrences. We will use the notation $A \geq B$ and $A \lesssim B$ to indicate that $A \geq C B$ and $A \leq C B$, respectively, where $A$ and $B$ are expressions depending on functions that in the discrete case may depend on $h$ as well. Analogously, $A \approx B$ will mean that $B \lesssim A \lesssim B$,

The following theorem is a simplified version of the corresponding one in Ref. [4].

Theorem 2.1. Stability of the continuous problem

For all $[u, p] \in V_{0} \times Q$ there exists $[v, q] \in V_{0} \times Q$ for which

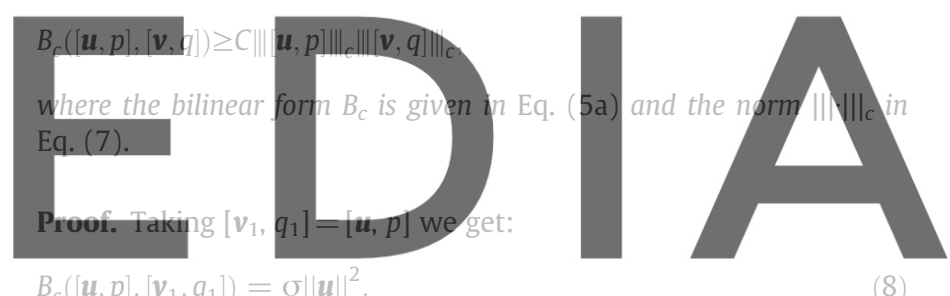

$\left.B_{c}\left([\boldsymbol{u}, p],\left[\boldsymbol{v}_{1}, q_{1}\right]\right)=\sigma \| \boldsymbol{u}\right]^{2}$

$(8)$

\section{$\forall p \in L^{2}(\Omega) \exists v_{p} \in H_{0}(\operatorname{div}, \Omega) \mid-\left(p, \nabla \cdot v_{p}\right) \gtrsim\|p\|\left(\frac{1}{L_{0}}\left\|v_{p}\right\|+\left\|\nabla \cdot v_{p}\right\|\right)$}

We can choose $\boldsymbol{v}_{p}$ such that

$\left\|\boldsymbol{v}_{p}\right\|+L_{0}\left\|\nabla \cdot \boldsymbol{v}_{p}\right\|=\frac{1}{\sigma L_{0}}\|p\|$,

which is a dimensionally consistent norm. Taking $\left[\boldsymbol{v}_{2}, q_{2}\right]=\left[\boldsymbol{v}_{p}, 0\right]$ we have:

$B_{c}\left([\boldsymbol{u}, p],\left[\boldsymbol{v}_{2}, q_{2}\right]\right) \gtrsim-\sigma\|\boldsymbol{u}\|_{H(\operatorname{div}, \Omega)}^{2}+\frac{1}{\sigma L_{0}^{2}}\|p\|^{2}$.

Since $\boldsymbol{u} \in V_{0}$, we have that $\nabla \cdot \boldsymbol{u} \in L^{2}(\Omega)$. For $\left[\boldsymbol{v}_{3}, q_{3}\right]=\left[\mathbf{0}, \sigma L_{0}^{2} \nabla \cdot \boldsymbol{u}\right]$ we get:

$B_{c}\left([\boldsymbol{u}, p],\left[\boldsymbol{v}_{3}, q_{3}\right]\right)=\sigma L_{0}^{2}\|\nabla \cdot \boldsymbol{u}\|^{2}$.

Let $[\boldsymbol{v}, q]=\sum_{i=1}^{3} \alpha_{i}\left[\boldsymbol{v}_{i}, q_{i}\right] \in V_{0} \times Q, \alpha_{i} \in \mathbb{R}$. The coefficients $\alpha_{i}$ can be chosen so that

$B_{c}([\boldsymbol{u}, p],[\boldsymbol{v}, q]) \gtrsim\|[\boldsymbol{u}, p]\|_{c}^{2}$.

It is easily checked that $\|[\boldsymbol{v}, q]\|_{c} \lesssim\|[\boldsymbol{u}, p]\|_{c}$ for any combination of coefficients $\alpha_{i} \in \mathbb{R}$. This proves the theorem.

Remark 2.1. The inf-sup condition of Theorem 2.1 leads to stability bounds for velocity and pressure provided the data are regular; that is to 
say, $L_{c}$ is continuous with respect to $\|\mid \cdot\|_{c}$. This continuity is true for $\boldsymbol{f} \in$ $H(\operatorname{div}, \Omega)^{\prime}$ and $g \in L^{2}(\Omega)$.

Remark 2.2. If there is more regularity of the data, that is, if $\boldsymbol{f} \in L^{2}(\Omega)^{d}$ and $g \in L^{2}(\Omega)$, the pressure belongs to $H^{1}(\Omega)$ and we can pose the problem in a different functional setting. Let now the pressure space be the closure of $C^{\infty}(\Omega) / \mathbb{R}$ with respect to $\left(\sigma L_{0}^{2}\right)^{-1 / 2}\|q\|+\sigma^{-1 / 2}\|\nabla q\|$, that reduces to $H^{1}(\Omega)$. We consider the following weak formulation: find $[\boldsymbol{u}, p] \in H_{0}(\operatorname{div}, \Omega) \times H^{1}(\Omega)$ (trial space) such that

$B_{c}([\boldsymbol{u}, p],[\boldsymbol{v}, q])=L_{c}([\boldsymbol{v}, q]), \quad \forall[\boldsymbol{v}, q] \in L^{2}(\Omega)^{d} \times L^{2}(\Omega)$.

Note that the trial and test spaces are different. Control over $\frac{1}{\sigma}\|\nabla p\|^{2}$ can be obtained by taking as test function in Eq. (5a) $\left[\boldsymbol{v}_{4}, q_{4}\right]=[\nabla p, 0] \in$ $L^{2}(\Omega)^{d} \times L^{2}(\Omega)$. Now, taking a linear combination of this test function and the test functions in the proof of Theorem 2.1, $[\boldsymbol{v}, q]=\sum_{i=1}^{4} \alpha_{i}\left[\boldsymbol{v}_{i}, q_{i}\right] \in$ $L^{2}(\Omega)^{d} \times L^{2}(\Omega)$, and picking appropriate coefficients $\alpha_{i} \in \mathbb{R}$, we get stability over $\|[\boldsymbol{u}, p]\|_{c}+\frac{1}{\sqrt{G}}\|\nabla p\|$. This is the functional setting in which stability of the continuous problem has been proved in Ref. [4]

\section{Non-conforming finite element approximation}

Let us introduce some notation. The FE partition will be denoted by $\mathcal{T}_{h}=\{K\}$, and summation over all the elements will be indicated by $\sum_{K}$. For conciseness, $\mathcal{T}_{h}=\{K\}$ will be assumed quasi-uniform, being $h$ the mesh size. The broken integral $\sum_{K} \int_{K}$ will be denoted by $\int_{\mathcal{T}_{h}}$. The collection of all edges (faces, for $d=3$ ) will be written as $\mathcal{E}_{h}=\{E\}$ and summation over all these edges will be indicated as $\sum_{F}$. The set of

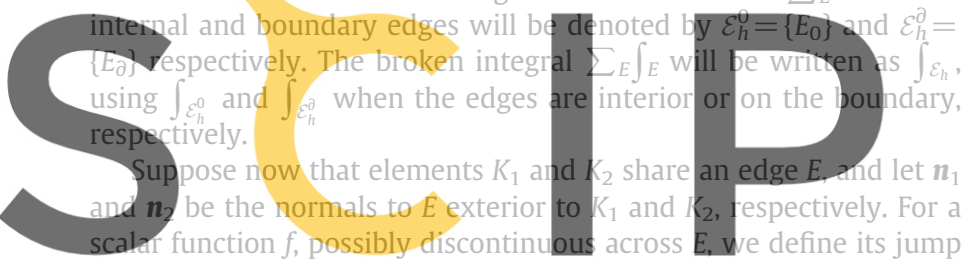
and average as

Register fơlerkfreemattahttetps//www.scipedia.com to d

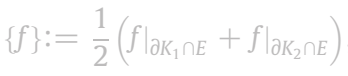

whereas for vectorial quantities we will use

$$
\begin{aligned}
& \llbracket \boldsymbol{v} \rrbracket:=\left.\boldsymbol{n}_{1} \cdot \boldsymbol{v}\right|_{\partial K_{1} \cap E}+\left.\boldsymbol{n}_{2} \cdot \boldsymbol{v}\right|_{\partial K_{2} \cap E}, \\
& \{\boldsymbol{v}\}:=\frac{1}{2}\left(\left.\boldsymbol{v}\right|_{\partial K_{1} \cap E}+\left.\boldsymbol{v}\right|_{\partial K_{2} \cap E}\right) .
\end{aligned}
$$

We extend these definitions on $\Gamma$ as $\llbracket f \rrbracket:=\boldsymbol{n} f$ and $\{f\}:=f$ and similarly for vector functions. Let us consider piecewise discontinuous FE spaces for the velocity and the pressure, given respectively by

$$
\begin{aligned}
& V_{h}:=\left\{\boldsymbol{v} \in\left(L^{2}(\Omega)\right)^{d}|\boldsymbol{v}|_{K} \in R_{k}(K)^{d} \forall K \in \mathcal{T}_{h}\right\}, \\
& Q_{h}:=\left\{q \in L^{2}(\Omega) / \mathbb{R}|q|_{K} \in R_{l}(K) \forall K \in \mathcal{T}_{h}\right\},
\end{aligned}
$$

where $R_{m}$ consists of polynomials in $x_{1}, \ldots, x_{d}$ of degree less than or equal to $m$ when $K$ is a simplex and of degree less than or equal to $m$ in each coordinate when $K$ is a quadrilateral (hexahedron, when $d=3$ ). Thus, $k$ and $l$ are the order of approximation of velocity and pressure, respectively. This is a non-conforming approximation of problem (4). The notion of non-conforming approximation depends on the way the continuous problem is posed. In particular, a discontinuous approximation of the velocity is not conforming for the first functional setting introduced above (because $V_{h} \not \subset H(\operatorname{div}, \Omega)$ ) whereas it is conforming in the mixed Laplacian setting. Similarly, if instead of using Eq. (4) the problem is posed using hybrid methods in which the continuity of the (a priori discontinuous) solution is enforced via Lagrange multipliers, a discontinuous approximation is conforming. In what follows, the concept of conforming (and subsequently nonconforming) approximation is considered with respect to the velocity-pressure space $H(\operatorname{div}, \Omega) \times L^{2}(\Omega)$. Likewise, we will use the term discontinuous Galerkin (dG) referring to the discontinuous functions in the interpolation spaces, even if the discrete formulations we will analyze are not of Galerkin type.

With the aim of obtaining a well-defined weak formulation of the continuous problem (1) for dG approximations, let us test Eqs. (1a) and (1b) against functions in $V_{h} \times Q_{h}{ }^{2}$ Taking the FE test functions $\left[\boldsymbol{v}_{h}^{K}, q_{h}^{K}\right]$ with support in an element $K$ and integrating some terms by parts, we obtain

$$
\begin{aligned}
& \int_{K} \sigma \boldsymbol{u} \cdot \boldsymbol{v}_{h}^{K} \mathrm{~d} \Omega-\int_{K} p \nabla \cdot \boldsymbol{v}_{h}^{K} \mathrm{~d} \Omega \\
&+\int_{\partial K} q_{h}^{K} \boldsymbol{n} \cdot \boldsymbol{u} \mathrm{d} \Gamma=\int_{K} \boldsymbol{f} \cdot \boldsymbol{v}_{h}^{K} \mathrm{~d} \Gamma-\int_{K} \mathrm{~d} \Omega+\int_{K} g q_{h}^{K} \mathrm{~d} \Omega
\end{aligned}
$$

The discontinuous FE space $V_{h} \times Q_{h}$ is spanned by discontinuous functions with support in a single element, so that for any $\left[v_{h}, q_{h}\right] \in$ $V_{h} \times Q_{h},\left[\boldsymbol{v}_{h}, q_{h}\right]=\sum_{K}\left[\boldsymbol{v}_{h}^{K}, q_{h}^{K}\right]$. Adding up Eq. (10) for all $K \in \mathcal{T}_{h}$, using formula

\section{$\sum_{K} \int_{\partial K} \phi \boldsymbol{n} \cdot \boldsymbol{w} \mathrm{d} \Gamma=\int_{\varepsilon^{0}} \llbracket \phi \rrbracket \cdot\{\boldsymbol{w}\} \mathrm{d} \Gamma+\int_{\varepsilon_{\mathrm{h}}} \llbracket \boldsymbol{w} \rrbracket\{\phi\} \mathrm{d} \Gamma$ $=\int_{\varepsilon_{h}} \llbracket \phi \rrbracket \cdot\{\boldsymbol{w}\} \mathrm{d} \Gamma+\int_{\varepsilon^{0}} \llbracket \boldsymbol{w} \rrbracket\{\phi\} \mathrm{d} \Gamma$}

invoking the continuity of velocities $\llbracket u \rrbracket=0$ and fluxes $\llbracket p \rrbracket=0$ for

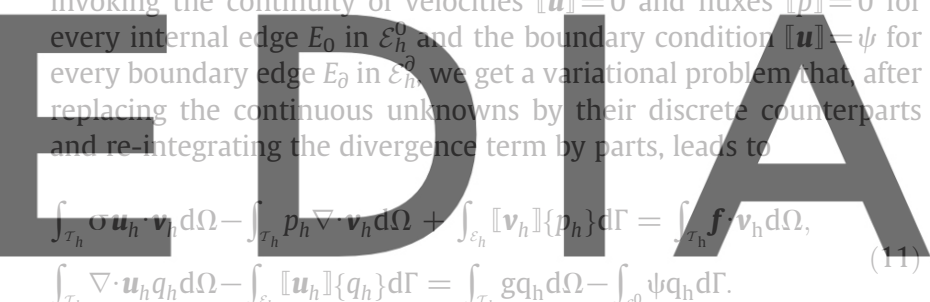

download the version without the watermark Consistently with the notation introduced above, the symbol $\left\langle f_{1}, f_{2}\right\rangle_{D}$ will be used to denote the integral of the product of functions $f_{1}$ and $f_{2}$ over $D$, with $D=K$ (an element), $D=\partial K$ (an element boundary) or $D=E$ (an edge). Likewise, $\left\|f_{1}\right\|_{D}^{2}:=\left\langle f_{1}, f_{1}\right\rangle_{D}$. With all these notations, let us write the problem in a compact manner, e.g. using the divergence form Eq. (11). It consists in finding $\left[\boldsymbol{u}_{h}, p_{h}\right] \in$ $V_{h} \times Q_{h}$ such that

$B_{d}\left(\left[\boldsymbol{u}_{h}, p_{h}\right],\left[\boldsymbol{v}_{h}, q_{h}\right]\right)=L_{d}\left(\left[\boldsymbol{v}_{h}, q_{h}\right]\right) \quad \forall\left[\boldsymbol{v}_{h}, q_{h}\right] \in V_{h} \times Q_{h}$,

where

$$
\begin{aligned}
B_{d}\left(\left[\boldsymbol{u}_{h}, p_{h}\right],\left[\boldsymbol{v}_{h}, q_{h}\right]\right)= & \sigma\left(\boldsymbol{u}_{h}, \boldsymbol{v}_{h}\right)-\sum_{K}\left\langle p_{h}, \nabla \cdot v_{h}\right\rangle_{K}+\sum_{K}\left\langle\nabla \cdot u_{h}, q_{h}\right\rangle_{K} \\
& +\sum_{E}\left\langle\left\{p_{h}\right\}, \llbracket \boldsymbol{v}_{h} \rrbracket\right\rangle_{E}-\sum_{E}\left\langle\left\{q_{h}\right\}, \llbracket \boldsymbol{u}_{h} \rrbracket\right\rangle_{E}, \\
L_{d}\left(\left[\boldsymbol{v}_{h}, q_{h}\right]\right)=\left\langle\boldsymbol{f}, \boldsymbol{v}_{h}\right\rangle+ & +\left\langle g, q_{h}\right\rangle-\sum_{E_{\partial}}\left\langle\psi, q_{h}\right\rangle_{E_{\partial}} .
\end{aligned}
$$

We have ended up with a FE formulation that allows us to use piecewise discontinuous functions; the continuity of normal velocities and pressures has already been enforced in a weak way, as well as the normal velocity boundary condition. Unfortunately, this formulation is not stable and the weak enforcement of normal velocity boundary conditions is too weak. In the next section we motivate stabilizing terms that lead to a well-posed discrete problem with a weak (but

\footnotetext{
${ }^{2}$ We cannot use Eq. (4) since $V_{h} \times Q_{h} \not \subset V \times Q$ in general.
} 
effective) enforcement of the normal trace of the velocity on the boundary.

\section{A stabilized finite element method}

In this section we introduce some stabilization techniques for the FE approximation of the Darcy problem. These stabilization techniques are motivated by the variational multiscale (VMS) framework introduced in Ref. [19]. The use of the VMS approach for the Darcy problem can also be found in Ref. [24]. Our approach is different to the one in these references; we motivate a different set of stabilization parameters and stabilization terms that open a new discussion, namely, the choice of the characteristic length. Different expressions for the length scales that appear in our stabilization parameters lead to a set of methods with different stability and convergence properties. We motivate methods that mimic both variational frameworks in Section 2 and some intermediate situations, whereas the approaches in Refs. [23,24] can only mimic the mixed Laplacian setting. Furthermore, we consider two different choices of the so-called subgrid proiection that are well-settled for the Stokes problem (see e.g. Refs. [10,19]).

We target a unified method that will accommodate continuous and discontinuous approximations. Therefore, the FE spaces for both velocity and pressure, denoted by $V_{h}$ and $Q_{h}$, respectively, are free to be either continuous (conforming) or discontinuous. In all cases, the stabilization methods can be stated as follows: find $\left[\boldsymbol{u}_{h}, p_{h}\right] \in V_{h} \times Q_{h}$ such that

$B_{s}\left(\left[\boldsymbol{u}_{h}, p_{h}\right],\left[\boldsymbol{v}_{h}, q_{h}\right]\right)=L_{s}\left(\left[\boldsymbol{v}_{h}, q_{h}\right]\right) \quad \forall\left[\boldsymbol{v}_{h}, q_{h}\right] \in V_{h} \times Q_{h}$.

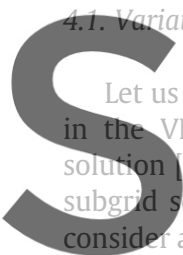
ional multiscale formulation

Let us start with a brief motivation Sontion $\left.u_{1}, p\right]$ of Ees: $(4)-(5)$ into its cale $\left[\boldsymbol{u}^{\prime}, p^{\prime}\right]$. In order to hav the moment, we consider $V_{h} \times Q_{h} \subset V \times Q$.

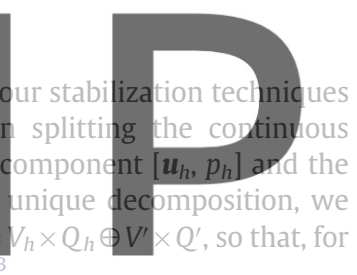

Invoking this decomposition in the continuous problem for both

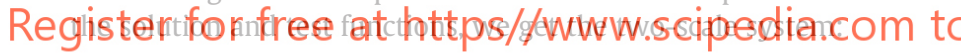

$$
\begin{aligned}
& B_{c}\left(\left[\boldsymbol{u}_{h}, p_{h}\right],\left[\boldsymbol{v}_{h}, q_{h}\right]\right)+B_{c}\left(\left[\boldsymbol{u}^{\prime}, p^{\prime}\right],\left[\boldsymbol{v}_{h}, q_{h}\right]\right)=L_{c}\left(\left[\boldsymbol{v}_{h}, q_{h}\right]\right), \\
& \boldsymbol{B}_{c}\left(\left[\boldsymbol{u}_{h}, \tilde{p}_{h}\right],\left[\boldsymbol{v}, q^{\prime}\right]\right)+\boldsymbol{B}_{c}\left(\left[\boldsymbol{u}^{\prime}, p^{\prime}\right],\left[\boldsymbol{v}^{\prime}, q^{\prime}\right]\right)=L_{c}\left(\left[\boldsymbol{v}^{\prime}, q^{\prime}\right]\right),
\end{aligned}
$$

for all $\left[\boldsymbol{v}_{h}, q_{h}\right] \in V_{h} \times Q_{h}$ and $\left[\boldsymbol{v}^{\prime}, q^{\prime}\right] \in V^{\prime} \times Q^{\prime}$. This is an infinitedimensional problem equivalent to Eqs. (4)-(5) that is unfeasible for numerical purposes. Further approximations must be considered in order to get a finite dimensional problem (see Refs. [3,10] for a very detailed exposition). After integration-by-parts of some terms, and assuming that the subgrid component can be localized inside every finite element, we get:

$$
\begin{aligned}
& B_{c}\left(\left[\boldsymbol{u}_{h}, p_{h}\right],\left[\boldsymbol{v}_{h}, q_{h}\right]\right)+\left\langle\left[\boldsymbol{u}^{\prime}, p^{\prime}\right], \mathcal{L}^{*}\left[\boldsymbol{v}_{h}, q_{h}\right]\right\rangle=L\left(\left[\boldsymbol{v}_{h}, q_{h}\right]\right), \\
& \mathcal{P}^{\prime}\left(\mathcal{L}\left[\boldsymbol{u}^{\prime}, p^{\prime}\right]\right)=\mathcal{P}^{\prime}\left([\boldsymbol{f}, g]-\mathcal{L}\left[\boldsymbol{u}_{h}, p_{h}\right]\right),
\end{aligned}
$$

where the operator $\mathcal{P}^{\prime}$ is the broken $L^{2}$-projection onto $V^{\prime}$ (see Section 4.2) and $\mathcal{L}^{*}$ is the adjoint of the Darcy operator $\mathcal{L}$, defined by $\mathcal{C}[\boldsymbol{u}, p]=$ $[\sigma \boldsymbol{u}+\nabla p, \nabla \cdot \boldsymbol{u}]$. The second term in Eq. (14a) is the stabilization term, whereas the second equation is the (still infinite-dimensional) subgrid equation. Obviously, the expression for the subscale is not exact, it is just an approximation of the exact problem, since some terms on element edges have been neglected. We refer to Ref. [14] for a discussion about the approximation of inter-element jumps. The next step consists in

\footnotetext{
${ }^{3}$ Let us note that the space $V \times Q$ does not include boundary conditions. This avoids the discussion about boundary conditions for the subscale. In any case, their inclusion would be straightforward, by splitting $\psi$ into a FE part that belongs to the space of traces of finite element functions and the respective sub-grid component.
}

replacing the differential operator $\mathcal{L}$ by an algebraic one. Inside every element, this operator is approximated by a matrix of stabilization parameters $\tau^{-1}$, and the subgrid projection $\mathcal{P}^{\prime}$ by an appropriate approximation $\mathcal{P}_{h}^{\prime}:=\left[\mathcal{P}_{h, u}^{\prime}, \mathcal{P}_{h, p}^{\prime}\right]$. Then, Eq. (14b) can be approximated by

$\tau^{-1}\left[\boldsymbol{u}^{\prime}, p^{\prime}\right]=\mathcal{P}_{h}^{\prime}\left([\boldsymbol{f}, g]-\mathcal{L}\left[\boldsymbol{u}_{h}, p_{h}\right]\right)$

from where the subscale component has a closed form in terms of the FE component. Let us assume the stabilization matrix to be a diagonal matrix $\tau=\operatorname{diag}\left(\tau_{u} \mathbf{I}, \tau_{p}\right)$, I being the $d \times d$ identity. In this case, we have

$$
\boldsymbol{u}^{\prime}=\tau_{u} P_{h, u}^{\prime}\left(\boldsymbol{f}-\sigma \boldsymbol{u}_{h}-\nabla p_{h}\right), \quad \quad p^{\prime}=\tau_{p} \mathcal{P}_{h, q}^{\prime}\left(g-\nabla \cdot \boldsymbol{u}_{h}\right) .
$$

Using these expressions for the subscales in the FE problem (14a), we get the stabilized versions of $B_{c}$ and $L_{c}$ :

$$
\begin{aligned}
B_{s c}\left(\left[\boldsymbol{u}_{h}, p_{h}\right],\left[\boldsymbol{v}_{h}, q_{h}\right]\right)= & B_{c}\left(\left[\boldsymbol{u}_{h}, p_{h}\right],\left[\boldsymbol{v}_{h}, q_{h}\right]\right)+\tau_{p} \sum_{K}\left\langle\mathcal{P}_{h, p}^{\prime}\left(\nabla \cdot \boldsymbol{u}_{h}\right), \nabla \cdot \boldsymbol{v}_{h}\right\rangle_{K} \\
& +\tau_{u} \sum_{K}\left\langle\mathcal{P}_{h, u}^{\prime}\left(\sigma \boldsymbol{u}_{h}+\nabla p_{h}\right),-\sigma \boldsymbol{v}_{h}+\nabla q_{h}\right\rangle_{K},
\end{aligned}
$$

$$
\begin{aligned}
L_{S C}\left(\left[\boldsymbol{v}_{h}, q_{h}\right]\right)= & L_{c}\left(\left[\boldsymbol{v}_{h}, q_{h}\right]\right)+\tau_{p} \sum_{K}\left\langle\mathcal{P}_{h, p}^{\prime}(g), \nabla \cdot \boldsymbol{v}_{h}\right\rangle_{K} \\
& +\tau_{u} \sum_{K}\left\langle\mathcal{P}_{h, u}^{\prime}(\boldsymbol{f}),-\sigma \boldsymbol{v}_{h}+\nabla q_{h}\right\rangle_{K} .
\end{aligned}
$$

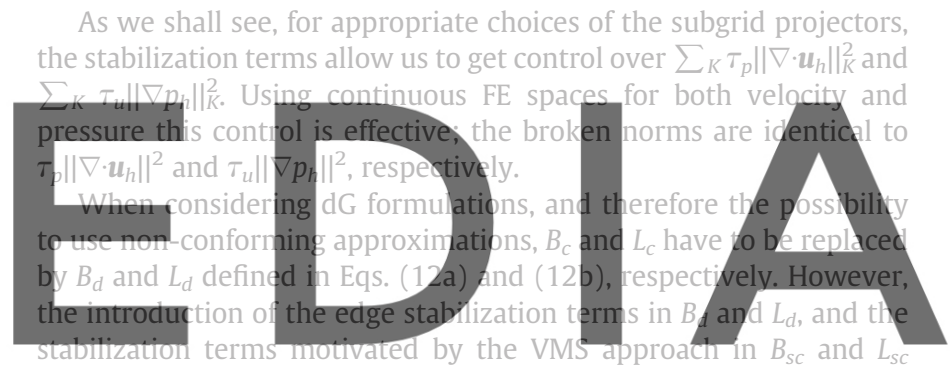

defined in Eqs. (15a) and (15b) are not enough because they only give

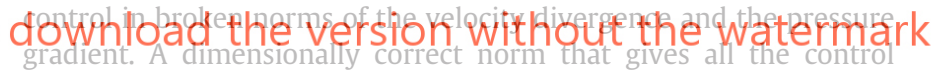
needed for discontinuous velocities is

$$
\sum_{K} \tau_{p}\left\|\nabla \cdot \boldsymbol{u}_{h}\right\|_{K}^{2}+\sum_{E} \frac{\tau_{p}}{h}\left\|\llbracket \boldsymbol{u}_{h} \rrbracket\right\|_{E}^{2}
$$

and analogously for the pressure

$$
\sum_{K} \tau_{u}\left\|\nabla p_{h}\right\|_{K}^{2}+\sum_{E} \frac{\tau_{u}}{h}\left\|\llbracket p_{h} \rrbracket\right\|_{E}^{2}
$$

In order to get stability in these norms, to account for nonconforming approximations and, at the same time, to incorporate non-homogeneous velocity boundary conditions $\boldsymbol{n} \cdot \boldsymbol{u}=\psi$ on $\Gamma$, we modify $B_{s c}$ to $B_{s}$ and $L_{s c}$ to $L_{s}$, defined respectively as

$$
\begin{aligned}
B_{s}\left(\left[\boldsymbol{u}_{h}, p_{h}\right],\left[\boldsymbol{v}_{h}, q_{h}\right]\right)= & B_{d}\left(\left[\boldsymbol{u}_{h}, p_{h}\right],\left[\boldsymbol{v}_{h}, q_{h}\right]\right) \\
& +\tau_{p} \sum_{K}\left\langle\mathcal{P}_{h, p}^{\prime}\left(\nabla \cdot \boldsymbol{u}_{h}\right), \nabla \cdot \boldsymbol{v}_{h}\right\rangle_{K} \\
& +\tau_{u} \sum_{K}\left\langle\mathcal{P}_{h, u}^{\prime}\left(\sigma \boldsymbol{u}_{h}+\nabla p_{h}\right),-\sigma \boldsymbol{v}_{h}+\nabla q_{h}\right\rangle_{K} \\
& +\frac{\tau_{p}}{h} \sum_{E}\left\langle\llbracket \boldsymbol{u}_{h} \rrbracket, \llbracket \boldsymbol{v}_{h} \rrbracket\right\rangle E+\frac{\tau_{u}}{h} \sum_{E_{0}}\left\langle\llbracket p_{h} \rrbracket, \llbracket q_{h} \rrbracket\right\rangle_{E_{0}},
\end{aligned}
$$

$$
\begin{aligned}
L_{s}\left(\left[\boldsymbol{v}_{h}, q_{h}\right]\right)= & L_{d}\left(\left[\boldsymbol{v}_{h}, q_{h}\right]\right)+\tau_{p} \sum_{K}\left\langle\mathcal{P}_{h, p}^{\prime}(g), \nabla \cdot \boldsymbol{v}_{h}\right\rangle_{K} \\
& +\tau_{u} \sum_{K}\left\langle\mathcal{P}_{h, u}^{\prime}(\boldsymbol{f}),-\sigma \boldsymbol{v}_{h}+\nabla q_{h}\right\rangle_{K}+\frac{\tau_{p}}{h} \sum_{E_{\partial}}\left\langle\psi, \llbracket \boldsymbol{v}_{h} \rrbracket\right\rangle_{E_{\partial}} .
\end{aligned}
$$


Here and below we have considered $\tau_{u}$ and $\tau_{p}$ constant for all the elements, in accordance with the assumption of quasi-uniformity of the family of finite element meshes.

It is easy to see that the last two terms in Eq. (16a) provide the desired control over the jumps. Furthermore, these terms are consistent, in the sense that they vanish when $\left[\boldsymbol{u}_{h}, p_{h}\right]$ is replaced by $[\boldsymbol{u}, p]$ (for sufficiently smooth $p$ ). Let us point out that the velocity boundary condition has already been enforced in a weak sense, à la Nitsche, with a penalty coefficient $\frac{\tau_{p}}{h}$ (see e.g. Ref. [26]). We refer to Ref. [14] for a different motivation of stabilizing jump terms based on the VMS decomposition.

We have ended up with a stabilized discrete problem for continuous and discontinuous FE approximations. The definition of $\tau$ is an essential ingredient of any stabilization technique, and in particular of this one. We motivate an expression for these parameters in the next subsection.

Remark 4.1. For the Darcy problem, the pressure subscale cannot be neglected, since the Galerkin terms do not control the velocity in $H($ div, $\Omega)$. At the continuous level, this stability can be understood in two different ways. One way is to note that the bilinear form for $u$ is coercive with respect to $H(\operatorname{div}, \Omega)$ when it is restricted to the kernel of the constraint. It can also be understood as a consequence of the additional inf-sup condition (6). Therefore, both velocity and pressure stability rely on inf-sup conditions. The Stokes problem is very different, since the bilinear form for the velocity is coercive in the whole velocity space; the pressure subscale can be neglected because the $H^{1}(\Omega)$ velocity stability comes from Galerkin terms.

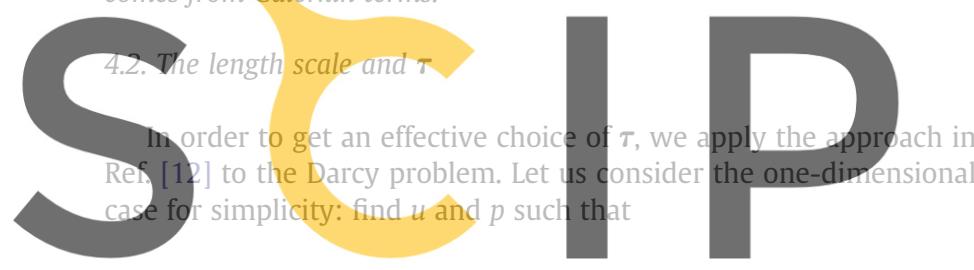

$\sigma u+p_{x}=f$

\section{Register fó $\overline{\bar{r}}$ free at https//www.scipedia.com to}

where the subscript $(\cdot)_{x}$ denotes the spatial derivative. Let $U=[u, p]$ be the unknown of the probiem and $F=[f, g]$ the force vector, and let $M$ be a positive definite matrix that defines a pointwise product in the space of admissible force vectors. Up to factors, the only diagonal matrix that defines a dimensionally correct inner product (all terms with the same dimensions) is:

$M=\left[\begin{array}{cc}\frac{1}{\sigma} & 0 \\ 0 & \sigma \ell^{2}\end{array}\right]$,

where $\ell$ has dimensions of length. This matrix defines the pointwise norm $|F|_{M}^{2}=F \cdot M F$. We will also make use of the norm $\|\left. F\right|_{K, M} ^{2}=\int_{K}|F|_{M}^{2} \mathrm{~d} \Omega$ restricted to an element $K$.

Since $U^{\prime}$ is the part of the solution that cannot be captured by the FE space, we assume that its Fourier transform is dominated by wave numbers of order $h^{-1} \tilde{k}$, where $\tilde{k}$ is an order $\mathcal{O}(1)$ dimensionless quantity. Therefore, the Fourier transform of $\mathcal{P}^{\prime}\left(\mathcal{L} U^{\prime}\right)$ inside an element $K$ (neglecting boundary values) can be approximated by $\mathcal{S}(\tilde{k}) \hat{U}^{\prime}$, where

$$
\mathcal{S}(\tilde{k})=\left[\begin{array}{cc}
\sigma & \frac{i \tilde{k}}{h} \\
\frac{\tilde{k}}{h} & 0
\end{array}\right],
$$

with $i=\sqrt{-1}$. Using Plancherel's formula we easily get

$$
\begin{aligned}
\left\|\mathcal{P}^{\prime}\left(\mathcal{L} U^{\prime}\right)\right\|_{K, M}^{2} & \approx \int\left|S(\tilde{k}) \hat{U}^{\prime}\right|_{K, M}^{2} \mathrm{~d} \tilde{k} \leq\left\|S\left(\tilde{k}_{0}\right)\right\|_{K, M}^{2}\left\|\hat{U}^{\prime}\right\|_{K, M^{-1}}^{2} \\
& \approx\|\mathcal{S}(\tilde{k} 0)\|_{K, M}^{2}\left\|U^{\prime}\right\|_{K, M^{-1}}^{2}
\end{aligned}
$$

where $\tilde{k}_{0}$ is a mean wave number whose existence is established by the mean value theorem and the symbol $\approx$ has been used because boundary terms have been disregarded.

We want our choice of $\tau^{-1}$ to be real, diagonal and spectrally similar to $\mathcal{S}\left(\tilde{k}_{0}\right)$. Let $\boldsymbol{\tau}=\operatorname{diag}\left(\tau_{u}, \tau_{p}\right)$. We require that

$\operatorname{spec}\left(\bar{S}\left(\tilde{k}_{0}\right)^{t} M S\left(\tilde{k}_{0}\right)\right) \approx \operatorname{spec}\left(\left(\tau^{-1}\right)^{t} M \tau^{-1}\right)$,

where the spectrum is computed with respect to matrix $M^{-1}$. The two eigenvalues $\lambda_{i}$ (for $\left.i=1,2\right)$ of $\bar{S}\left(\tilde{k}_{0}\right)^{t} M S\left(\tilde{k}_{0}\right)$ that satisfy

$\operatorname{det}\left(\bar{S}\left(\tilde{k}_{0}\right)^{t} M S\left(\tilde{k}_{0}\right)-\lambda_{i} M^{-1}\right)=0$

are
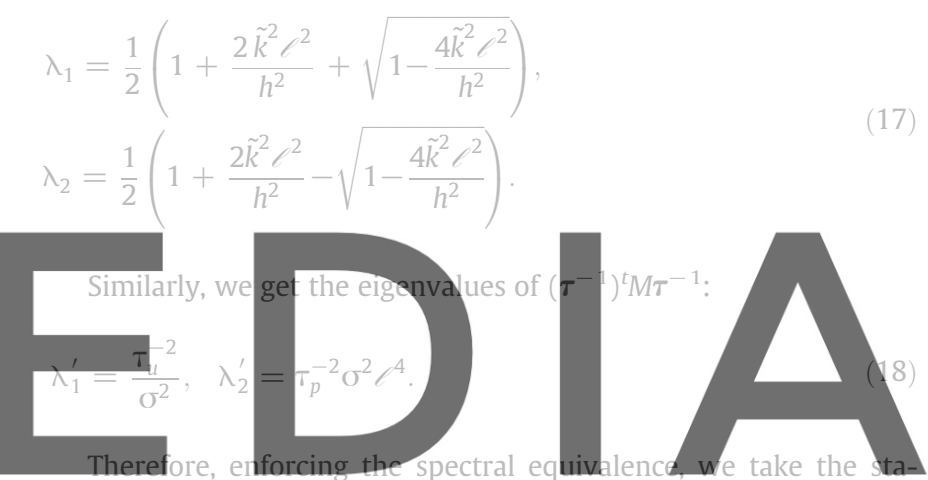

bilization parameters as

download the version without the watermark $\overline{\sigma \sqrt{\lambda_{1}}}, \tau_{p}=\frac{}{\sqrt{\lambda_{2}}}$.

In order to simplify the expression for the eigenvalues, we consider their asymptotic regime when $h \rightarrow 0$. In this situation, the components of $\tau$ can be written as

$\tau_{u}=\frac{h^{2}}{\sigma \ell_{u}^{2}}, \quad \tau_{p}=\sigma \ell_{p}^{2}$,

where $\ell_{u}$ and $\ell_{p}$ are parameters with dimension of length. Taking $\ell_{u}=h^{\alpha_{u}} L_{0}^{1-\alpha_{u}}$ and $\ell_{p}=h^{\alpha_{p}} L_{0}^{1-\alpha_{p}}$, with $\alpha_{u}, \alpha_{p} \in[0,1]$, we obtain a continuous range of finite element formulations that go from a method that mimics the primal mixed formulation (for $\alpha_{u}=1$ and $\alpha_{p}=1$ ) to another that mimics the dual mixed formulation (for $\alpha_{u}=0$ and $\alpha_{p}=0$ ). We will perform the numerical analysis for arbitrary length scales $\ell_{u}$ and $\ell_{p}$ but we will discuss with more detail and analyze using numerical experiments four different cases that correspond to the following choices:

- Method A: $\ell_{u}=c_{u} h$ and $\ell_{p}=c_{p} h$. In this case, the scaling is meshdependent, and gives

$\tau_{u} \sim \frac{1}{\sigma}, \quad \tau_{p} \sim \sigma h^{2}$

This method mimics the primal mixed formulation.

- Method B: $\ell_{u}=c_{u} L_{0}^{1 / 2} h^{1 / 2}$ and $\ell_{p}=c_{p} L_{0}^{1 / 2} h^{1 / 2}$, where $L_{0}$ is a characteristic length of the problem under consideration. This 
implies an a priori scaling of the continuous problem that leads to

$$
\tau_{u} \sim \frac{h}{\sigma L_{0}}, \quad \tau_{p} \sim \sigma L_{0} h
$$

This method consists of an intermediate situation between the primal and dual formulations.

- Method C: $\ell_{u}=c_{u} L_{0}$ and $\ell_{p}=c_{p} L_{0}$, again a mesh-dependent scaling. In this case, we get

$$
\tau_{u} \sim \frac{h^{2}}{\sigma L_{0}^{2}}, \quad \tau_{p} \sim \sigma L_{0}^{2}
$$

This method mimics the dual mixed formulation.

- Method D: $\ell_{u}=c_{u} h$ and $\ell_{p}=c_{u} L_{0}$, that leads to

$$
\tau_{u} \sim \frac{h}{\sigma L_{0}}, \quad \tau_{p} \sim \sigma L_{0}^{2}
$$

This method exhibits the stability properties of the continuous problem with regular data, namely $\boldsymbol{f} \in L^{2}(\Omega)^{d}$ and $g \in L^{2}(\Omega)$ (see Remark 2.2).

In these expressions, $c_{u}$ and $c_{p}$ are algorithmic dimensionless constants.

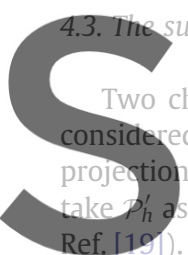
ubgrid projection

wo choices of the approximated onsidered (see Ref. [21] for a discl rojection based on the $H^{1}$-inner product) Assuming this, we end up with a stabilized method that we call algebraic subgrid scale (ASGS) method. Invoking the closed form of the subgrid scale in terms of the FE component, we get the following Registerforfofneerat: https//www.scipedia.com to

$$
\begin{aligned}
B_{S}\left(\left[\boldsymbol{u}_{h}, p_{h}\right],\left[v_{h}, q_{h}\right]\right)= & B_{d}\left(\left[\boldsymbol{u}_{h}, p_{h}\right],\left[v_{h}, q_{h}\right]\right)+\tau_{p} \sum_{K}\left\langle\nabla \cdot \boldsymbol{u}_{h}, \nabla \cdot v_{h}\right\rangle_{K} \\
& +\tau_{u} \sum_{K}\left\langle\sigma \boldsymbol{u}_{h}+\nabla p_{h},-\sigma \boldsymbol{v}_{h}+\nabla q_{h}\right\rangle_{K} \\
& +\frac{\tau_{p}}{h} \sum_{E}\left\langle\llbracket \boldsymbol{u}_{h} \rrbracket, \llbracket \boldsymbol{v}_{h} \rrbracket\right\rangle_{E}+\frac{\tau_{u}}{h} \sum_{E}\left\langle\llbracket p_{h} \rrbracket, \llbracket q_{h} \rrbracket\right\rangle_{E_{0}},
\end{aligned}
$$

$$
\begin{aligned}
L_{s}\left(\left[\boldsymbol{v}_{h}, q_{h}\right]\right)= & L_{d}\left(\left[\boldsymbol{v}_{h}, q_{h}\right]\right)+\tau_{p} \sum_{K}\left\langle g, \nabla \cdot \boldsymbol{v}_{h}\right\rangle_{K}+\tau_{u} \sum_{K}\left\langle\boldsymbol{f},-\sigma \boldsymbol{v}_{h}+\nabla q_{h}\right\rangle_{K} \\
& +\frac{\tau_{p}}{h} \sum_{E_{\partial}}\left\langle\psi, \llbracket \boldsymbol{v}_{h} \rrbracket\right\rangle_{E_{\partial}}
\end{aligned}
$$

To define the second subgrid projector, let us introduce some additional ingredients. Given a function $g$ such that $\left.g\right|_{K} \in L^{2}(K)$ for any element $K \in \mathcal{T}_{h}$, the broken $L^{2}$-projection over a Hilbert space $X$, denoted by $\Pi_{X}(g)$, is defined as the solution of:

$$
\left(\Pi_{X}(g), v\right)=\sum_{K}(g, v)_{K}, \quad \forall v \in X
$$

We also define $\Pi_{X}^{\perp}(g)=g-\Pi_{X}(g) \in L^{2}(\Omega)$. Using this notation, we define the orthogonal projection $\mathcal{P}_{h}^{\prime}([x, y]):=\left[\Pi_{V_{h}}^{\perp}(x), \Pi_{Q_{h}}^{\perp}(y)\right]$. This method is called as orthogonal subgrid scales method (see e.g. Ref. [10]). This choice is in concordance with the VMS decomposition, because the subgrid velocity component belongs to a subgrid space $V^{\prime}$ that satisfies $V^{\prime} \cap V_{h}=\{\mathbf{0}\}$. Let us note that the ASGS method does not necessarily satisfy this property for the Darcy problem. Again, writing the problem in terms of the FE component only, $B_{S}$ and $L_{S}$ for the OSS formulation read as follows:

$$
\begin{aligned}
& B_{s}\left(\left[\boldsymbol{u}_{h}, p_{h}\right],\left[\boldsymbol{v}_{h}, q_{h}\right]\right)= B_{d}\left(\left[\boldsymbol{u}_{h}, p_{h}\right],\left[\boldsymbol{v}_{h}, q_{h}\right]\right)+\tau_{p} \sum_{K}\left\langle\Pi_{Q_{h}}^{\perp}\left(\nabla \cdot \boldsymbol{u}_{h}\right), \nabla \cdot \boldsymbol{v}_{h}\right\rangle_{K} \\
&+\tau_{u} \sum_{K}\left\langle\Pi_{V_{h}}^{\perp}\left(\nabla p_{h}\right), \nabla q_{h}\right\rangle_{K}+\frac{\tau_{p}}{h} \sum_{E}\left\langle\llbracket \boldsymbol{u}_{h} \rrbracket, \llbracket \boldsymbol{v}_{h} \rrbracket\right\rangle_{E} \\
&+\frac{\tau_{u}}{h} \sum_{E}\left\langle\llbracket p_{h} \rrbracket, \llbracket q_{h} \rrbracket\right\rangle_{E}, \\
& L_{s}\left(\left[\boldsymbol{v}_{h}, q_{h}\right]\right)=L_{d}\left(\left[\boldsymbol{v}_{h}, q_{h}\right]\right)+\tau_{p} \sum_{K}\left\langle\Pi_{Q_{h}}^{\perp}(g), \nabla \cdot \boldsymbol{v}_{h}\right\rangle_{K} \\
&+\tau_{u} \sum_{K}\left\langle\Pi_{V_{h}}^{\perp}(\boldsymbol{f}), \nabla q_{h}\right\rangle_{K}+\frac{\tau_{p}}{h} \sum_{E_{\partial}}\left\langle\psi, \llbracket \boldsymbol{v}_{h} \rrbracket\right\rangle_{E_{\partial}} .
\end{aligned}
$$

The set of stabilization parameters designed in the previous section can be applied to both the ASGS and the OSS methods. Therefore, we have ended up with a number of methods, depending on the choice of the lengths $\ell_{u}$ and $\ell_{p}$ and the subgrid projection. In the next section we analyze the stability and convergence properties in all these cases. Finally, let us remark that in case of using continuous FE approximations, we recover a stabilized conforming formulation with Nitsche's enforcement of the normal trace of the velocity on the boundary.

Remark 4.2. Given $\boldsymbol{v}_{h} \in V_{h}$, if $\sigma v_{h} \notin V_{h}, \Pi_{V_{h}}\left(\sigma v_{h}\right) \neq 0$. However, using the non-consistent approach, we can still neglect this term without spoiling the accuracy.

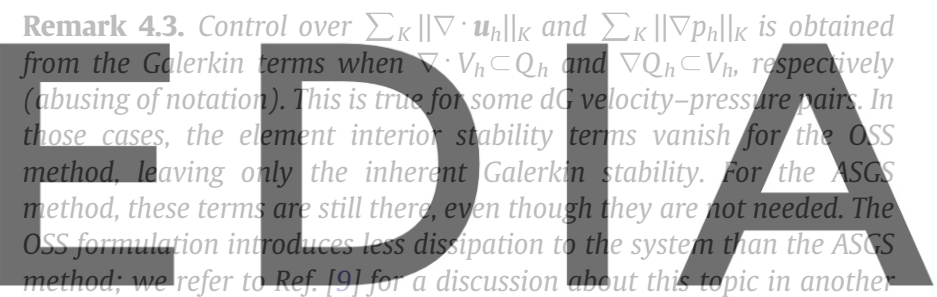

setting, when using conforming approximations.

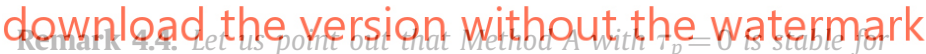

discontinuous Galerkin approximations, without the need of any extra jump term. The inherent stability mechanism that makes it possible has been analyzed in Ref. [6].

\section{Analysis of stabilized formulations for discontinuous approximations}

Let us introduce the mesh dependent norms

$$
\begin{aligned}
\left\|\left[\boldsymbol{v}_{h}, q_{h}\right]\right\|_{h}^{2}= & \sigma\left\|\boldsymbol{v}_{h}\right\|^{2}+\sigma \ell_{p}^{2} \sum_{K}\left\|\nabla \cdot \boldsymbol{v}_{h}\right\|_{K}^{2}+\frac{\sigma \ell_{p}^{2}}{h} \sum_{E}\left\|\llbracket \boldsymbol{v}_{h}\right\| \|_{E}^{2} \\
& +\frac{h^{2}}{\sigma \ell_{u}^{2}} \sum_{K}\left\|\nabla q_{h}\right\|_{K}^{2}+\frac{h}{\sigma \ell_{u}^{2}} \sum_{E}\left\|\llbracket q_{h}\right\| \|_{E}^{2}, \\
\left\|\left[\boldsymbol{v}_{h}, q_{h}\right]\right\| \|^{2}= & \left\|\left[\boldsymbol{v}_{h}, q_{h}\right]\right\|_{h}^{2}+\frac{1}{\sigma L_{0}^{2}}\left\|q_{h}\right\|^{2} .
\end{aligned}
$$

These are the norms in which the numerical analysis will be performed for both the ASGS and the OSS methods.

We define the interpolation error function

$E_{I}(h)^{2}=\sigma \ell_{p}^{2}\left(h^{-2} \varepsilon_{0}^{2}(\boldsymbol{u})+\varepsilon_{1}^{2}(\boldsymbol{u})\right)+\sigma \varepsilon_{0}^{2}(\boldsymbol{u})+\frac{h^{2}}{\sigma \ell_{u}^{2}}\left(h^{-2} \varepsilon_{0}^{2}(p)+\varepsilon_{1}^{2}(p)\right)$.

where, given a function $g, \varepsilon_{i}(g)=\left\|g-\tilde{g}_{h}\right\|_{H^{i}(\Omega)}$ and $\tilde{g}_{h}$ is an optimal FE interpolant of $g$. It will be proved that this is precisely the error function in the previous norm of the formulations introduced. 
Let us recall that we will consider for the sake of conciseness quasiuniform FE partitions (for the analysis of a stabilized formulation in the more general non-degenerate case, see Ref. [11]). Therefore, we assume that there is a constant $C_{\text {inv }}$, independent of the mesh size $h$ (the maximum of all the element diameters), such that

$\left\|\nabla v_{h}\right\|_{K} \leq C_{\text {inv }} h^{-1}\left\|v_{h}\right\|_{K}, \quad\left\|\Delta v_{h}\right\|_{K} \leq C_{\text {inv }} h^{-1}\left\|\nabla v_{h}\right\|_{K}$,

for all FE functions $v_{h}$ defined on $K \in \mathcal{T}_{h}$. This inequality can be used for scalars, vectors or tensors. Similarly, the trace inequality

$\|v\|_{\partial K}^{2} \leq C_{\operatorname{tr}}\left(h^{-1}\|v\|_{K}^{2}+h\|\nabla v\|_{K}^{2}\right)$

is assumed to hold for functions $v \in H^{1}(K), K \in \mathcal{T}_{h}$. If $\psi_{h}$ is a piecewise (continuous or discontinuous) polynomial, the last term in the previous inequality can be dropped using an inverse inequality, getting $\left\|\psi_{h}\right\|_{\partial K}^{2} \lesssim h^{-1}\left\|\psi_{h}\right\|_{K}^{2}$.

Using Eq. (24), for a given function $g$ we have that:

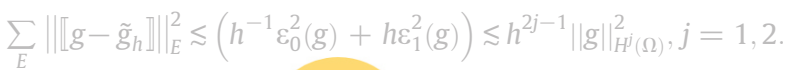

\section{Analogously, for a continuous function $g$ it holds:}

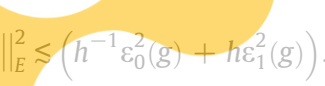

$\sum_{E}$

5.1. Analysis of the OSS method

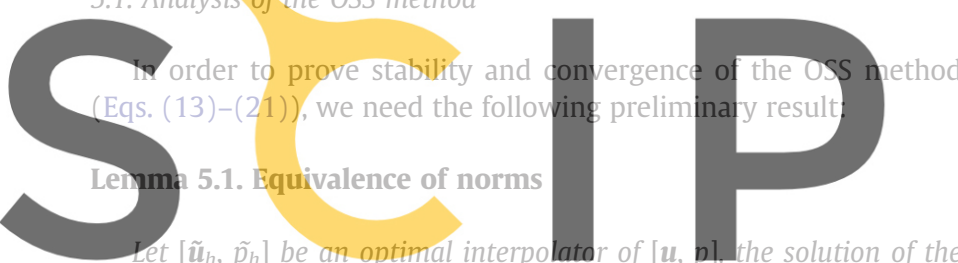
continuous problem (Eqs. (4)-(5)). Let $\left[\boldsymbol{u}_{h}, p_{h}\right]$ be the solution of the OSS stabilized FE problem (Eqs. (13)-(21)). Then, assuming that $k \geq 1$, the Registerviforefree ad dhttos//www.scipedia.com to

$$
\begin{gathered}
\left\|\left[\boldsymbol{u}_{h}, p_{h}\right]\right\| \lesssim\left\|\left[\boldsymbol{u}_{h}, p_{h}\right]\right\|\left\|_{h},\right\|\left[\tilde{\boldsymbol{u}}_{h}-\boldsymbol{u}_{h}, \tilde{p}_{h}-p_{h}\right]\|\lesssim\|\left[\tilde{u}_{h}-\boldsymbol{u}_{h}, \tilde{p}_{h}-p_{h}\right] \|_{h} \\
+E_{I}(h)+E_{C}(h) .
\end{gathered}
$$

Proof. From the inf-sup condition in the continuous case, for all $p \in L^{2}(\Omega)$ there exists a $\boldsymbol{v}_{p} \in H_{0}^{1}(\Omega)^{d}$ such that:

$$
\left(p, \nabla \cdot \boldsymbol{v}_{p}\right) \gtrsim \frac{1}{\sqrt{\sigma} L_{0}}\|p\|\left(\sqrt{\sigma}\left\|\boldsymbol{v}_{p}\right\|+\sqrt{\sigma} L_{0}\left\|\nabla \boldsymbol{v}_{p}\right\|\right),
$$

with $\left\|\boldsymbol{v}_{p}\right\|_{1}=\frac{1}{\sigma L_{0}}\|p\|$, where we consider a dimensionally consistent norm $\|\boldsymbol{v}\|_{1}:=\|\boldsymbol{v}\|+L_{0}\|\nabla \boldsymbol{v}\|$. Then, for $p_{h}$ there exists $\boldsymbol{v}_{p}$ for which

$$
\begin{aligned}
\frac{1}{\sigma L_{0}^{2}}\left\|p_{h}\right\|^{2} \lesssim & \left(p_{h}, \nabla \cdot \boldsymbol{v}_{p}\right)=\left(p_{h}, \nabla \cdot \tilde{\boldsymbol{v}}_{p, h}\right)-\sum_{K}\left\langle\nabla p_{h}, \boldsymbol{v}_{p}-\tilde{\boldsymbol{v}}_{p, h}\right\rangle_{K} \\
& +\sum_{E_{0}}\left\langle\llbracket p_{h} \rrbracket,\left\{\boldsymbol{v}_{p}-\tilde{\boldsymbol{v}}_{p, h}\right\}\right\rangle_{E_{0}}+\sum_{E_{0}}\left\langle\left\{p_{h}\right\}, \llbracket \boldsymbol{v}_{p}-\tilde{\boldsymbol{v}}_{p, h} \rrbracket\right\rangle_{E_{0}},
\end{aligned}
$$

where $\tilde{\boldsymbol{v}}_{p, h}$ is the Scott-Zhang interpolation ${ }^{4}$ of $\boldsymbol{v}_{p}$ onto $V_{h} \cap H_{0}^{1}(\Omega)$. Therefore, $\tilde{\boldsymbol{v}}_{p, h} \in \mathcal{C}^{0}(\Omega)$, and $k \geq 1$ is required (where $k$ is the order of the velocity FE space). In any case, $k \geq 1$ is needed for proving

\footnotetext{
${ }^{4}$ We explicitly consider this interpolation since the Scott-Zhang operator preserves homogeneous boundary conditions and it is a projection (see e.g. Ref. [18]). It allows us to use integration by parts without the introduction of terms on $\partial \Omega$.
}

convergence. We note that $\left[\left[\boldsymbol{v}_{p}\right]\right]=0$ and $\left[\left[\tilde{\boldsymbol{v}}_{p, h}\right]\right]=0$ on the set of edges $\mathcal{E}_{h}$. Using the interpolation property $\left\|\boldsymbol{v}_{p}-\tilde{\boldsymbol{v}}_{p, h}\right\| \lesssim \frac{h}{L_{0}}\left\|v_{p}\right\|_{1}$ and the fact that $h \lesssim \ell_{u} \lesssim L_{0}$, we get for the second term in the right-hand side of Eq. (26):

$-\sum_{K}\left\langle\nabla p_{h}, \boldsymbol{v}_{p}-\tilde{\boldsymbol{v}}_{p, h}\right\rangle_{K} \lesssim \sum_{K} \frac{h}{\sqrt{\sigma} \ell_{u}}\left\|\nabla p_{h}\right\|_{K} \frac{1}{\sqrt{\sigma} L_{0}}\left\|p_{h}\right\|$.

Using the trace inequality Eq. (24) and the $H^{1}$-continuity of the Scott-Zhang projector, we obtain for the edge terms:

$$
\begin{aligned}
& \sum_{E_{0}}\left\langle\llbracket p_{h} \rrbracket,\left\{\boldsymbol{v}_{p}-\tilde{\boldsymbol{v}}_{p, h}\right\}\right\rangle_{E_{0}} \lesssim \sum_{E_{0}} \frac{h^{1 / 2}}{\sqrt{\sigma} \ell}\left\|\llbracket p_{h} \rrbracket\right\|_{E_{0}} \frac{1}{\sqrt{\sigma} L_{0}}\left\|p_{h}\right\|, \\
& \sum_{E_{0}}\left\langle\left\{p_{h}\right\}, \llbracket \boldsymbol{v}_{p}-\tilde{\boldsymbol{v}}_{p, h} \rrbracket\right\rangle_{E_{0}}=0 .
\end{aligned}
$$

Finally, testing Eq. (21a) with $\left[\boldsymbol{v}_{h}, q_{h}\right]=\left[\tilde{\boldsymbol{v}}_{p, h}, 0\right]$ and using the fact that $h \lesssim \ell_{p} \lesssim L_{0}$ and $\left\|v_{h}\right\| \leq\left\|v_{h}\right\|_{1}$, we get:

$$
\begin{aligned}
\left(p_{h}, \nabla \cdot \tilde{\boldsymbol{v}}_{p, h}\right) & =\sigma\left(\boldsymbol{u}_{h}, \tilde{\boldsymbol{v}}_{p, h}\right)+\sigma \ell_{p}^{2}\left(\Pi_{Q_{h}}^{\perp}\left(\nabla \cdot \boldsymbol{u}_{h}\right), \nabla \cdot \tilde{\boldsymbol{v}}_{p, h}\right) \\
& \lesssim\left(\sqrt{\sigma}\left\|\boldsymbol{u}_{h}\right\|+\sqrt{\sigma} \ell_{p}\left\|\Pi_{Q_{h}}^{\perp}\left(\nabla \cdot \boldsymbol{u}_{h}\right)\right\|\right) \frac{1}{\sqrt{\sigma} L_{0}}\left\|p_{h}\right\| .
\end{aligned}
$$

With these ingredients, we prove the first part of the lemma. For the second part, the only difference is the control over the last term. Taking $v_{p}$ such that $\left\|v_{p}\right\|_{1}=\frac{1}{\sigma L}\left\|\tilde{p}_{h}-p_{h}\right\|$, we proceed as above, the

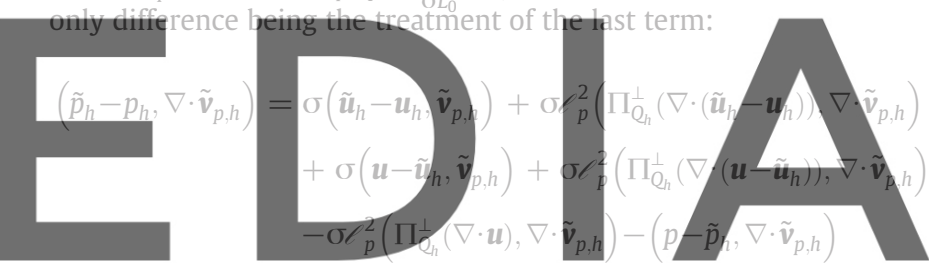

\section{download the version without the watermark}

\section{This proves the lemma.}

In the next theorem, we prove the stability properties of the OSS method in the working norms defined above. The OSS technique leads to a stabilized method that satisfies a discrete inf-sup condition and gives control over the velocity and pressure approximations in appropriate norms. The proof is constructive in the sense that we build a test function that implies the inf-sup condition.

\section{Theorem 5.1. (Stability)}

Let $\left[\boldsymbol{u}_{h}, p_{h}\right]$ be the solution of the OSS stabilized FE problem (Eqs. (13)-(21)). Then, the bilinear form $B_{s}$ satisfies a discrete inf-sup condition

$\inf _{\left[\boldsymbol{u}_{h}, p_{h}\right] \in V_{h} \times Q_{h}} \sup _{\left.\boldsymbol{v}_{h}, q_{h}\right] \in V_{h} \times Q_{h}} \frac{B_{S}\left(\left[\boldsymbol{u}_{h}, p_{h}\right],\left[\boldsymbol{v}_{h}, q_{h}\right]\right)}{\left\|\left[\boldsymbol{u}_{h}, p_{h}\right]\right\|\|\|\left\|\left[\boldsymbol{v}_{h}, q_{h}\right]\right\| \|_{h}} \geq \beta$.

In particular, for $k \geq 1$

$B_{s}\left(\left[\boldsymbol{u}_{h}, p_{h}\right], \Lambda\left(\left[\boldsymbol{u}_{h}, p_{h}\right]\right)\right) \geq\left.\left\|\left[\boldsymbol{u}_{h}, p_{h}\right]\right\|\right|^{2}$,

with

$\Lambda\left(\left[\boldsymbol{u}_{h}, p_{h}\right]\right)=\left[\boldsymbol{u}_{h}+\alpha \frac{h^{2}}{\sigma \ell_{u}^{2}} \Pi_{V_{h}}\left(\nabla p_{h}\right), p_{h}+\beta \sigma \ell_{p}^{2} \Pi_{Q_{h}}\left(\nabla \cdot \boldsymbol{u}_{h}\right)\right]$,

for $\alpha, \beta$ small enough constants that depend on $C_{\mathrm{inv}}$ and $C_{\mathrm{tr}}$. 
Proof. Stability is proved in three steps. First, taking $\boldsymbol{v}_{h}=\boldsymbol{u}_{h}$ and $q_{h}=p_{h}$ we obtain

$$
\begin{aligned}
& B_{S}\left(\left[\boldsymbol{u}_{h}, p_{h}\right],\left[\boldsymbol{u}_{h}, p_{h}\right]\right)=\sigma\left\|\boldsymbol{u}_{h}\right\|^{2}+\sigma \ell_{p}^{2}\left\|\Pi_{Q_{h}}^{\perp}\left(\nabla \cdot \boldsymbol{u}_{h}\right)\right\|^{2}+\frac{h^{2}}{\sigma \ell_{u}^{2}}\left\|\Pi_{V_{h}}^{\perp}\left(\nabla p_{h}\right)\right\|^{2} \\
& \quad+\frac{\sigma \ell_{p}^{2}}{h} \sum_{E}\left\|\llbracket \boldsymbol{u}_{h} \rrbracket\right\|_{E}^{2}+\frac{h}{\sigma \ell_{u}^{2}} \sum_{E_{0}}\|\| p_{h}\|\|_{E_{0}}^{2}=:\left\|\left[\boldsymbol{u}_{h}, p_{h}\right]\right\| \|_{*}^{2} .
\end{aligned}
$$

Now, taking $\left[\boldsymbol{v}_{h}, q_{h}\right]=\left[\mathbf{0}, \sigma \ell_{p}^{2} \Pi_{Q_{h}}\left(\nabla \cdot \boldsymbol{u}_{h}\right)\right]$ we get

$$
\begin{gathered}
B_{s}\left(\left[\boldsymbol{u}_{h}, p_{h}\right],\left[0, \ell_{p}^{2} \Pi_{Q_{h}}\left(\nabla \cdot u_{h}\right)\right]\right) \geq \sigma \ell_{p}^{2}\left\|\Pi_{Q_{h}}\left(\nabla \cdot u_{h}\right)\right\|^{2} \\
-c \frac{h}{\sqrt{\sigma} \ell}\left\|\Pi_{V_{h}}^{\perp}\left(\nabla p_{h}\right)\right\| \sqrt{\sigma} \ell_{p}\left\|\Pi_{Q_{h}}\left(\nabla \cdot \boldsymbol{u}_{h}\right)\right\| \\
-\left.c \frac{h^{1 / 2}}{\sqrt{\sigma} \ell} \sum_{E_{0}}\left\|\left[p_{h}\right]\right\|\right|_{E_{0}} \sqrt{\sigma} \ell_{p}\left\|\Pi_{Q_{h}}\left(\nabla \cdot u_{h}\right)\right\| \\
-c \frac{\sqrt{\sigma} \ell p}{h^{1 / 2}} \sum_{E}\left\|\llbracket \boldsymbol{u}_{h}\right\|\left\|_{E} \sqrt{\sigma} \ell p p \Pi_{Q_{h}}\left(\nabla \cdot \boldsymbol{u}_{h}\right)\right\| \\
\geq \frac{\sigma \ell_{p}^{2}}{2}\left\|\Pi_{Q_{h}}\left(\nabla \cdot u_{h}\right)\right\|^{2}-\frac{1}{4 \alpha}\left\|\left[u_{h}, p_{h}\right]\right\|_{*}^{2}
\end{gathered}
$$

for an appropriate constant $\alpha$, where we have used the assumption $\ell_{p} \lesssim \ell_{u}$. Now, let us consider the gradient form of the stabilized momentum equation, which is obtained by using
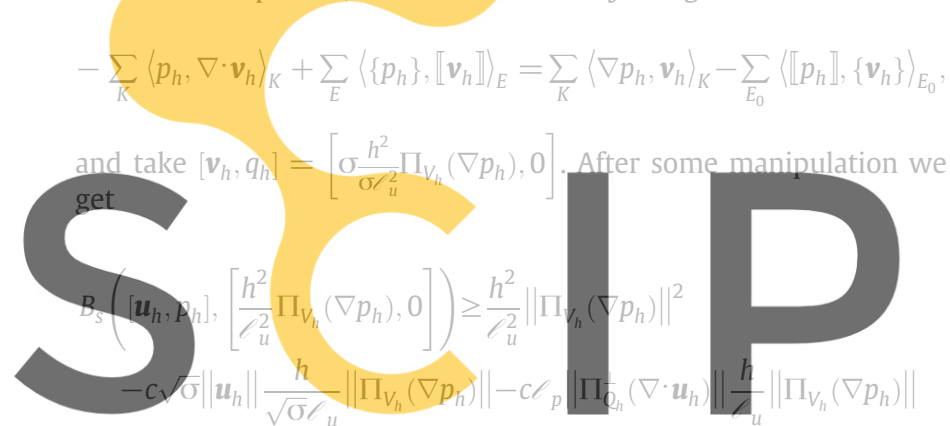

\section{$h^{1 / 2} \sum\left\|\left[p_{h}\right]\right\| \stackrel{h}{h}\left\|\Pi_{v}\left(\nabla p_{h}\right)\right\|_{0}$}

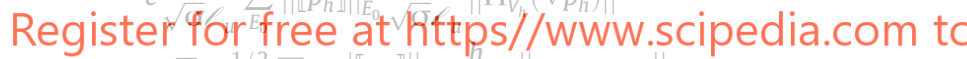

$$
-c \sqrt{\sigma} h^{-1 / 2} \sum_{E} l_{p}\left|\left\lfloor u_{h}\right]\left\|\left.\right|_{E} \frac{{ }_{h}}{\sqrt{\sigma} \ell_{u}}|| \Pi_{V_{h}}\left(\nabla p_{h}\right)\right\|\right.
$$

$$
\geq \frac{h^{2}}{2 \ell_{u}^{2}}\left\|\Pi_{V_{h}}\left(\nabla p_{h}\right)\right\|^{2}-\frac{1}{4 \beta}\left\|\left[\boldsymbol{u}_{h}, p_{h}\right]\right\|_{*}^{2}
$$

for an appropriate constant $\beta$, where we have used the fact that $h \lesssim \ell_{u}$. Combining all these results we get

$B_{s}\left(\left[\boldsymbol{u}_{h}, p_{h}\right], \Lambda\left(\left[\boldsymbol{u}_{h}, p_{h}\right]\right)\right) \geq 2\left\|\left[\boldsymbol{u}_{h}, p_{h}\right]\right\|_{h}^{2}$.

In order to prove the theorem, we need the continuity of $\Lambda$, that is to say, $\left\|\left|\Lambda\left(\left[\boldsymbol{u}_{h}, p_{h}\right]\right)\left\||| \lesssim\left|\left\|\left[\boldsymbol{u}_{h}, p_{h}\right] \mid\right\|\right.\right.\right.\right.$. It is easily seen that

$$
\begin{aligned}
\| \Lambda & \left.\Lambda\left(\boldsymbol{u}_{h}, p_{h}\right]\right)\|\|^{2} \lesssim\left\|\left[\boldsymbol{u}_{h}, p_{h}\right]\right\|\left\|^{2}+\frac{h^{4}}{\sigma \ell_{u}^{4}}\right\| \Pi_{V_{h}}\left(\nabla p_{h}\right) \|^{2} \\
& +\frac{h^{4} \ell_{p}^{2}}{\sigma \ell_{u}^{4}} \sum_{K}\left\|\nabla \cdot \Pi_{V_{h}}\left(\nabla p_{h}\right)\right\|_{K}^{2}+\frac{h^{3} \ell_{p}^{2}}{\sigma \ell_{u}^{4}} \sum_{E}\left\|\llbracket \Pi_{V_{h}}\left(\nabla p_{h}\right) \rrbracket\right\|_{E}^{2} \\
& +\frac{\sigma \ell_{p}^{4}}{L_{0}^{2}}\left\|\Pi_{Q_{h}}\left(\nabla \cdot \boldsymbol{u}_{h}\right)\right\|^{2}+\frac{\sigma \ell_{p}^{4} h^{2}}{\ell_{u}^{2}} \sum_{K}\left\|\nabla \cdot \Pi_{Q_{h}}\left(\nabla \cdot \boldsymbol{u}_{h}\right)\right\|_{K}^{2} \\
& \left.+\frac{\sigma \ell_{p}^{4} h}{\ell_{u}^{2}} \sum_{E} \| \llbracket \Pi_{Q_{h}}\left(\nabla \cdot \boldsymbol{u}_{h}\right)\right]\left\|_{E}^{2} \lesssim\right\|\left[\boldsymbol{u}_{h}, p_{h}\right]\left\|^{2}+\frac{1}{\sigma L_{0}^{2}}\right\| q_{h} \|^{2},
\end{aligned}
$$

where we have used inverse inequalities, trace inequalities, and the relations

$h \lesssim \ell_{p} \lesssim \ell_{u} \lesssim L_{0}$.
Analogously, we get $\left\|\left|\Lambda\left(\left[\boldsymbol{u}_{h}, p_{h}\right]\right)\right|\right\|_{h} \lesssim\left\|||\left[\boldsymbol{u}_{h}, p_{h}\right]\right\| \|_{h}$. All these results are not only true for $\left[\boldsymbol{u}_{h}, p_{h}\right]$ but for any FE function in $V_{h} \times Q_{h}$. From Eq. (27) and using the continuity of $\Lambda(\cdot)$ for the norm $\|\mid \cdot\| \|_{h}$ we get the inf-sup condition. Using the previous lemma and Eq. (27) we prove the second part of the theorem.

From this theorem we conclude that the OSS technique leads to a stable method in the working norms (22). Let us stress the fact that the stability constant $\beta$ does not depend on physical and numerical parameters. On the other hand, the norm in which the stability is proved mimics the continuous solution. So, stability is effective for any value of $\sigma$, and mimics the stability bound satisfied by the exact solution. This is particularly important for flow in porous media, since $\sigma$ can take values that go from $10^{8}$ to $10^{-6}$.

In order to prove the accuracy of the algorithm, let us bound the interpolation error:

\section{Lemma 5.2. (Interpolation error)}

Let $[u, p]$ be the solution of the continuous problem (Eqs. (4)-(5)) and $\left[\tilde{\boldsymbol{u}}_{h}, \tilde{p}_{h}\right]$ an optimal interpolator in $V_{h} \times Q_{h}$. We also assume that the length scales in the stabilization parameters satisfy $\ell_{u} \leqslant \ell_{p}$. Then, the following interpolation error estimate holds:

$B_{S}\left(\left[\boldsymbol{u}-\tilde{\boldsymbol{u}}_{h}, p-\tilde{p}_{h}\right],\left[\boldsymbol{v}_{h}, q_{h}\right]\right) \leq E_{I}(h)\left\|\left[\boldsymbol{v}_{h}, q_{h}\right]\right\|_{h}$

Proof. The symmetric terms can be easily bounded by using the Cauchy-Schwarz inequality. The rest of the terms can be bounded as
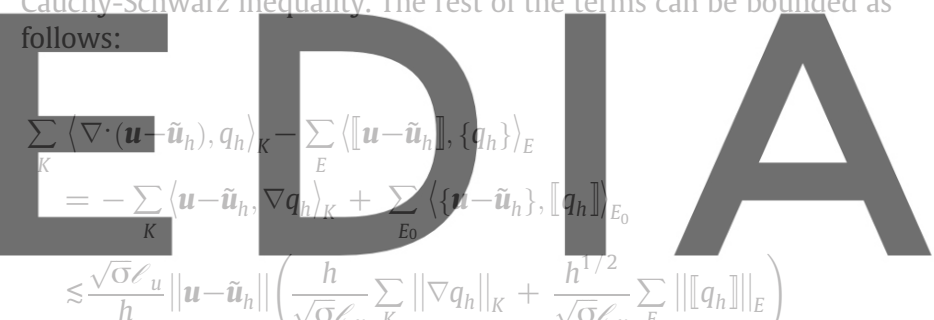

$\leq \frac{\sqrt{\sigma} / u}{h}\left\|u-\tilde{u}_{h}\right\|\left(\frac{h}{\sqrt{\sigma} / l_{u}} \sum_{K_{0}}\left\|\nabla q_{h}\right\|_{K}+\frac{h^{1 / 2}}{\sqrt{\sigma} /{ }_{u}} \sum_{E}\left\|\llbracket q_{h}\right\| \|_{E}\right)$

download the version without the watermark

$\lesssim \frac{{ }_{p} p}{h} \varepsilon_{0}(\boldsymbol{u})\left\|\left[v_{q}, q_{h}\right]\right\|$

$$
\begin{aligned}
& -\sum_{K}\left\langle p-\tilde{p}_{h}, \nabla \cdot \boldsymbol{v}_{h}\right\rangle_{K}+\sum_{E}\left\langle\left\{p-\tilde{p}_{h}\right\}, \llbracket v_{h} \rrbracket\right\rangle_{E} \\
& \quad \lesssim \frac{1}{\sqrt{\sigma} \ell_{p}} \sum_{K}\left\|p-\tilde{p}_{h}\right\|_{K}\left(\sqrt{\sigma} \ell p p \mid \nabla \cdot \boldsymbol{v}_{h}\left\|+\sum_{E} \frac{\sqrt{\sigma} \ell p}{h^{1 / 2}}\right\| \llbracket \boldsymbol{v}_{h} \rrbracket\|\|_{E}\right) \\
& \quad \lesssim \frac{1}{\sqrt{\sigma} \ell_{u}} \varepsilon_{0}(p)\left\|\left[\boldsymbol{v}_{h}, q_{h}\right]\right\|_{h} .
\end{aligned}
$$

Using the definition of $\mathrm{E}_{I}(h)$ (Eq. (23)) we finish the proof of the lemma.

Using the stability properties in Theorem 5.1 and the bound for the interpolation error in Lemma 5.2, we can prove the following convergence result:

\section{Theorem 5.2. (Convergence)}

Let $[\boldsymbol{u}, p]$ be the solution of the continuous problem (Eqs. (4)-(5)) and let $\left[\boldsymbol{u}_{h}, p_{h}\right]$ be the solution of the OSS stabilized FE problem (Eqs. (13)(21)). We also assume that the length scales in the stabilization parameters satisfy $\ell_{u}=\ell_{p}$ and $k \geq 1$. Then, the following error estimate holds:

$\left\|\left[\boldsymbol{u}-\boldsymbol{u}_{h}, p-p_{h}\right]\right\| \lesssim\left(E_{I}(h)+E_{C}(h)\right)$. 
Proof. Let $\left[\tilde{\boldsymbol{u}}_{h}, \tilde{p}_{h}\right]$ be an optimal interpolator of $[\boldsymbol{u}, p]$ in $V_{h} \times Q_{h}$. From the previous results it follows that

$$
\begin{aligned}
& \left\|\left[\tilde{\boldsymbol{u}}_{h}-\boldsymbol{u}_{h}, \tilde{p}_{h}-p_{h}\right]\right\|\|\|_{h}\left\|\left[\boldsymbol{v}_{h}, q_{h}\right]\right\| \|_{h} \lesssim B_{s}\left(\left[\tilde{\boldsymbol{u}}_{h}-\boldsymbol{u}, \tilde{p}_{h}-p_{h}\right],\left[\boldsymbol{v}_{h}, q_{h}\right]\right) \\
& \quad \lesssim B_{s}\left(\left[\tilde{\boldsymbol{u}}_{h}-\boldsymbol{u}, \tilde{p}_{h}-p_{h}\right],\left[\boldsymbol{v}_{h}, q_{h}\right]\right)+B_{s}\left(\left[\boldsymbol{u}-\boldsymbol{u}_{h}, p-p_{h}\right],\left[\boldsymbol{v}_{h}, q_{h}\right]\right) \\
& \quad \lesssim\left(E_{I}(h)+E_{C}(h)\right)\left\|\left[\boldsymbol{v}_{h}, q_{h}\right]\right\| \|_{h},
\end{aligned}
$$

where $\left[\boldsymbol{v}_{h}, q_{h}\right]$ is chosen so that Theorem 5.1 holds. We conclude the proof using the second result in Lemma 5.1, the triangle inequality and the fact that $\left\|\left|\left[\boldsymbol{u}-\tilde{\mathbf{u}}_{h}, p-\tilde{p}_{h}\right] \|\right| \leqslant E_{I}(h)\right.$.

Remark 5.1. For the OSS stabilization technique, $\ell_{p} \lesssim \ell_{u}$ is needed for stability and $\ell_{u} \lesssim \ell_{p}$ for convergence, so that we require $\ell_{p} \approx \ell_{u}$. Therefore, the choice of the stabilization parameters in Method $D$ with the OSS stabilized system (Eqs. (13)-(21)) is out of this analysis.

\subsection{Analysis of the ASGS method}

The stability and convergence analysis for the ASGS method is similar to the one for the OSS formulation, but not identical. The main difference, as we will show below, is the different nature of the stability in every case. As in the previous section, let us start with the relation between the two working norms for the FE solution and interpolation error.

Lemma 5.3. Equivalence of norms

Let $\left[\tilde{\boldsymbol{u}}_{h}, \tilde{p}_{h}\right]$ be an optimal interpolator of $[\boldsymbol{u}, p]$, the solution of the

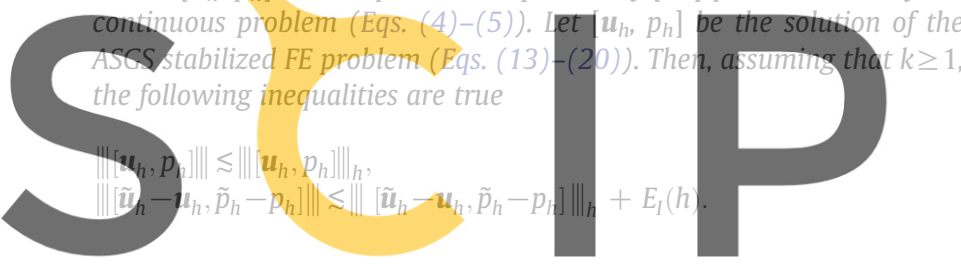

Proof. The proof only differs from the one for the OSS method in

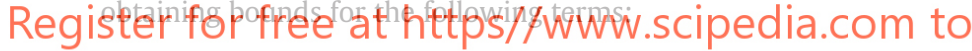

$$
\begin{aligned}
\left(p_{h}, \nabla \cdot \tilde{v}_{p}, h\right)= & \sigma\left(\boldsymbol{u}_{h}, \tilde{v}_{p, h}\right)+\sigma \ell_{p}^{2} \sum_{K}\left\langle\nabla \cdot \boldsymbol{u}_{h}, \nabla \cdot \tilde{v}_{p, h}\right\rangle_{K} \\
& +\frac{h^{2}}{\sigma \ell_{u}^{2}} \sum_{K}\left\langle\boldsymbol{\sigma} \boldsymbol{u}_{h}+\nabla p_{h},-\sigma \tilde{\boldsymbol{v}}_{p, h}\right\rangle_{K} \lesssim\left\|\left[\boldsymbol{u}_{h}, p_{h}\right]\right\|_{h} \frac{1}{\sigma L_{0}}\left\|p_{h}\right\|,
\end{aligned}
$$

where we have used that $h \lesssim \ell u$, and

$$
\begin{aligned}
\left(\tilde{p}_{h}-p_{h}, \nabla \cdot \tilde{\boldsymbol{v}}_{p, h}\right)= & \sigma\left(\tilde{\boldsymbol{u}}_{h}-\boldsymbol{u}_{h}, \tilde{\boldsymbol{v}}_{p, h}\right)+\sigma \ell_{p}^{2} \sum_{K}\left\langle\nabla \cdot\left(\tilde{\boldsymbol{u}}_{h}-\boldsymbol{u}_{h}\right), \nabla \cdot \tilde{\boldsymbol{v}}_{p, h}\right\rangle_{K} \\
& +\frac{h^{2}}{\sigma \ell_{u}^{2}} \sum_{K}\left\langle\sigma\left(\tilde{\boldsymbol{u}}_{h}-\boldsymbol{u}_{h}\right)+\nabla\left(\tilde{p}_{h}-p_{h}\right),-\sigma \tilde{\boldsymbol{v}}_{p, h}\right\rangle_{K} \\
& +\sigma\left(\boldsymbol{u}-\tilde{\boldsymbol{u}}_{h}, \tilde{\boldsymbol{v}}_{p, h}\right)+\sigma \ell_{p}^{2} \sum_{K}\left\langle\nabla \cdot\left(\boldsymbol{u}-\tilde{\boldsymbol{u}}_{h}\right), \nabla \cdot \tilde{\boldsymbol{v}}_{p, h}\right\rangle_{K} \\
& +\frac{h^{2}}{\sigma \ell_{u}^{2}} \sum_{K}\left\langle\sigma\left(\boldsymbol{u}-\tilde{\boldsymbol{u}}_{h}\right)+\nabla\left(p-\tilde{p}_{h}\right),-\sigma \tilde{\boldsymbol{v}}_{p, h}\right\rangle_{K} \\
\lesssim & \left(\left\|\left[\tilde{\boldsymbol{u}}_{h}-\boldsymbol{u}_{h}, \tilde{p}_{h}-p_{h}\right]\right\|_{h}+E_{I}(h)\right) \frac{1}{\sigma L_{0}}\left\|\tilde{p}_{h}-p_{h}\right\|,
\end{aligned}
$$

from where the second part of the Theorem follows.

In the next theorem, we prove the coercivity of $B_{S}$ for the ASGS stabilization.

\section{Theorem 5.3. (Stability)}

Let $\left[\boldsymbol{u}_{h}, p_{h}\right]$ be the solution of the ASGS stabilized FE problem (Eqs. (13)-(20)) with a choice of the length scales that satisfies $\ell_{p} \leqslant \ell_{u}$. Let us also assume that the algorithmic constant in the definition of $\uparrow_{u}$ is $c_{u}>1$ and that $k \geq 1$. Then, the bilinear form $B_{s}$ satisfies the coercivity property

$B_{S}\left(\left[\boldsymbol{u}_{h}, p_{h}\right],\left[\boldsymbol{u}_{h}, p_{h}\right]\right) \geq\left\|\left[u_{h}, p_{h}\right]\right\|^{2}$.

Proof. For the ASGS method, stability is simply proved taking $\left[\boldsymbol{v}_{h}, q_{h}\right]=$ $\left[\boldsymbol{u}_{h}, p_{h}\right]:$

$$
\begin{aligned}
B_{s}\left(\left[\boldsymbol{u}_{h}, p_{h}\right],\left[\boldsymbol{u}_{h}, p_{h}\right]\right)= & \left(1-\frac{1}{c_{u}^{2}}\right) \sigma\left\|\boldsymbol{u}_{h}\right\|^{2}+\sigma \ell_{p}^{2} \sum_{K}\left\|\nabla \cdot \boldsymbol{u}_{h}\right\|_{K}^{2} \\
& +\frac{h^{2}}{\sigma \ell_{u}^{2}} \sum_{K}\left\|\nabla p_{h}\right\|_{K}^{2}+\left.\frac{\sigma \ell_{p}^{2}}{h}\left\|\left.\llbracket \llbracket \boldsymbol{u}_{h} \rrbracket\right|_{\varepsilon_{h}} ^{2}+\frac{h}{\sigma \ell_{u}^{2}}\right\| \llbracket p_{h} \rrbracket\right|_{\varepsilon_{h}^{0}} ^{2} .
\end{aligned}
$$

The first term in the right-hand side of this equality is positive under the assumption that $c_{u}>1$, that implies $h<\ell_{u}$.

The previous theorem proves that the ASGS technique leads to a positive definite bilinear form, whereas the OSS technique leads to a bilinear form that satisfies a discrete inf-sup condition (see Ref. [131), that is to say, $B_{S}$ is an indefinite bilinear form, as its continuous counterpart $B_{c}$. This is an essential difference between both stabilization techniques that makes the analysis of the OSS method slightly more involved. However, the lack of coercivity for the OSS approach is not a drawback at all; the stabilized problem in this case only introduces what is not controlled by the Galerkin terms and inherits the stability mechanism of the continuous problem.

In order to prove convergence results, let us start bounding the interpolation error:

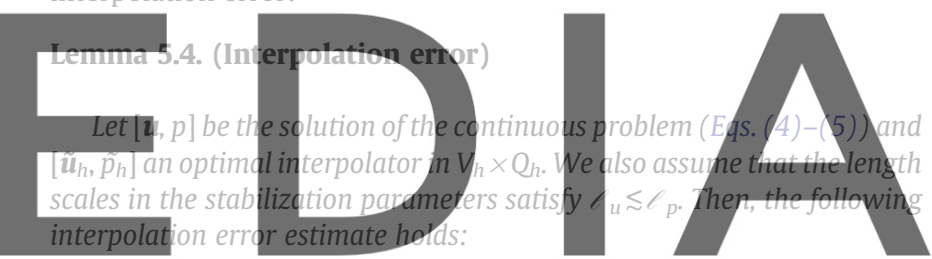

Proof. All the terms can be easily bounded by using the CauchySchwarz inequality and the bounds proved in Lemma 5.2 for the OSS method.

The convergence result for this algorithm is stated in the following theorem:

\section{Theorem 5.4. (Convergence)}

Let $[\boldsymbol{u}, p]$ be the solution of the continuous problem (Eqs. (4)-(5)) and $\left[\tilde{\boldsymbol{u}}_{h}, \tilde{p}_{h}\right]$ an optimal interpolator in $V_{h} \times Q_{h}$. Let $\left[\boldsymbol{u}_{h}, p_{h}\right]$ be the solution of the ASGS stabilized FE problem (Eqs. (13)-(20)). We also assume that the length scales in the stabilization parameters satisfy $\ell_{u} \leq \ell_{p}$ and $k \geq 1$. Then, the following error estimate holds:

$\left\|\left[\boldsymbol{u}-\boldsymbol{u}_{h}, p-p_{h}\right]\right\| \leqslant E_{I}(h)$.

The proof is very similar to the one for Theorem 5.2 and has been omitted.

Remark 5.2. For the ASGS method, the assumption $\ell_{p} \lesssim \ell_{u}$ is not needed. Therefore, the previous result applies for Method $D$ introduced earlier. Let us remark that $\ell_{u} \leqslant \ell_{p}$ is still needed for convergence. It does not allow us to take $\ell_{u}=c_{u} L_{0}$ and $\ell_{p}=c_{p} h$.

In any case, both the ASGS and the OSS algorithms lead to the same orders of convergence. Another important aspect of this analysis is the effect of the stabilization parameters in the stability and convergence results. We will discuss this effect in Section 7. 


\section{Duality arguments and improved convergence estimates}

In the previous section a priori error estimates have been obtained for both the ASGS and the OSS methods. For conforming FE approximations of the velocity, sharper error estimates in $L^{2}(\Omega)$ for

$$
\boldsymbol{e}_{u}=\boldsymbol{u}-\boldsymbol{u}_{h}, \quad e_{p}=p-p_{h}
$$

have been obtained by the authors in Ref. [4] by using Aubin-Nitschetype duality arguments. These results are obtained assuming that the adjoint problem

$$
\begin{array}{lc}
\sigma \boldsymbol{w}-\nabla \xi=\sigma \boldsymbol{f} & \text { in } \Omega, \\
-\nabla \cdot \boldsymbol{w}=\frac{1}{\sigma L_{0}^{2}} g & \text { in } \Omega, \\
\boldsymbol{n} \cdot \boldsymbol{w}=0 & \text { in } \Gamma,
\end{array}
$$

satisfies the elliptic regularity assumptions

$$
\begin{array}{ll}
\|\xi\|_{2} \lesssim \frac{1}{L_{0}^{2}}\|g\|+\sigma\|\nabla \cdot \boldsymbol{f}\| & \text { if } \boldsymbol{f} \in H(\operatorname{div}, \Omega), \\
\|\boldsymbol{w}\|_{1} \lesssim \frac{1}{\sigma L_{0}^{2}}\|g\| & \text { if } \boldsymbol{f}=0,
\end{array}
$$

together with the obvious general stability estimate

$$
\|\boldsymbol{w}\| \leq\|\boldsymbol{f}\| \quad \text { if } g=0 \text {. }
$$

It is known that Eqs. (28) and (29) hold if $\Omega$ is convex and polyhedral or with twice differentiable boundary. The improved error estimate for the pressure is obtained in Ref. [4] taking $\boldsymbol{f}=\mathbf{0}$ and $g=e_{p}$. Therefore, since $e_{p} \in L^{2}(\Omega)$, the regularity assumptions (28) and (29) can be used. For the sharper velocity estimates we should take $\boldsymbol{f}=\boldsymbol{e}_{u}$ and $g=0$. Since $\nabla \cdot \boldsymbol{e}_{u}$ does not belong to $L^{2}(\Omega)$ for velocity approximations that are not conforming in $H(\operatorname{div}, \Omega)$, Eq. (28) is meaningless and the classical AubinNitsche-type duality arguments do not apply.

The error estimates obtained in Theorems 5.2-5.4 can be written as

$$
\begin{aligned}
\sigma\left\|\boldsymbol{e}_{u}\right\|^{2}+\sigma \ell_{p}^{2} \sum_{K}\left\|\nabla \cdot \boldsymbol{e}_{u}\right\|_{K}^{2}+\frac{\sigma \ell_{p}^{2}}{h} \sum_{E}\left\|\llbracket \boldsymbol{e}_{u} \rrbracket\right\|_{E}^{2} \\
+\frac{1}{\sigma L_{0}^{2}}\left\|e_{p}\right\|^{2}+\frac{h^{2}}{\sigma \ell_{u}^{2}} \sum_{K}\left\|\nabla e_{p}\right\|_{K}^{2}+\frac{h}{\sigma \ell_{u}^{2}} \sum_{E}\left\|\llbracket e_{p} \rrbracket\right\|_{E}^{2} \\
\quad \leq \sigma \ell_{p}^{2} h^{2 k}\|\boldsymbol{u}\|_{k+1}^{2}+\sigma h^{2 k+2}\|\boldsymbol{u}\|_{k+1}^{2}+\frac{1}{\sigma \ell_{u}^{2}} h^{2 l+2}\|p\|_{l+1}^{2}
\end{aligned}
$$

Using duality arguments for the OSS method, we get improved error estimates for the pressure in the next theorem.

Theorem 6.1. Assume the same conditions as in Theorem 5.2 and, moreover, assume Eqs. (28) and (29) to hold. Furthermore, for $\ell_{u}=h$ and piecewise constant pressures $(l=0)$ we also require the constant $c_{u}$ in Section 4.2 to be large enough. Under these assumptions, there holds

$\left\|e_{p}\right\|^{2} \lesssim \sigma^{2} \ell_{p}^{4}\left\|\nabla \cdot \boldsymbol{e}_{u}\right\|^{2}+h^{2} \sum_{K}\left\|\nabla e_{p}\right\|_{K}^{2}$.

When $V_{h} \subset \mathcal{C}^{0}(\Omega)$, we also have:

$\left\|\boldsymbol{e}_{u}\right\|^{2} \lesssim\left(h^{2}+\frac{\ell_{p}^{4}}{L_{0}^{2}}+h^{2} \frac{\ell_{p}^{4}}{\ell_{u}^{4}}\right)\left\|\nabla \cdot \boldsymbol{e}_{u}\right\|^{2}+\frac{1}{\sigma^{2}}\left(\frac{h^{4}}{\ell_{u}^{4}}+\frac{h^{2}}{L_{0}^{2}}\right) \sum_{K}\left\|\nabla e_{p}\right\|_{K}^{2}$,

Proof. We have assumed that the order of the piecewise polynomial functions that span $V_{h}$ are of order greater or equal to one $(k \geq 1)$, that is to say, piecewise constant velocity approximations cannot be used. Thanks to that, we can pick an optimal FE interpolant $\tilde{\boldsymbol{w}}_{h}$ of $\boldsymbol{w}$ such that $\tilde{w}_{h} \in V_{h} \cap H^{1}(\Omega)^{d}$. Therefore, all the terms involving jumps of $\boldsymbol{w}$ and $\tilde{\boldsymbol{w}}_{h}$ cancel. At this point, the proof of the improved error estimate over the pressure follows the one for conforming FE approximations for the velocity, that can be found in Ref. [4].

Let us use the same duality arguments for the ASGS method.

Theorem 6.2. Assume the same conditions as in Theorem 5.4 and, moreover, assume Eqs. (28) and (29) to hold. Furthermore, for $\ell_{u}=h$ and piecewise constant pressures $(l=0)$ we also require the constant $c_{u}$ in Section 4.2 to be large enough. For $l>1$, we simply require $c_{u}>1$. Under these assumptions, Eq. (32) holds. When $V_{h} \subset \mathcal{C}^{0}(\Omega)$, Eq. (33) is also true.

Proof. Again, we note that $w$ can be approximated by a $\mathcal{C}^{0} \mathrm{FE}$ interpolant that belongs to $V_{h}$. Therefore, the proof in Ref. [4] for continuous FE velocity spaces can be extended to dG approximations. $\square$

\section{The right choice of $\ell_{u}$ and $\ell_{p}$}

In the previous section we have proved the error estimate Eq. (31) with respect to what could be called the energy norm of the stabilized methods. An improved bound Eq. (32) for $\left\|e_{p}\right\|$ has been obtained using duality arguments. This estimate is always true for methods B and C; when piecewise constant pressures are used together with methods $\mathrm{A}$ and $\mathrm{D}$ this result only holds for $c_{u}$ large enough. The sharper bound for $\left\|\boldsymbol{e}_{u}\right\|$ in Eq. (33) is only true for conforming approximations; it does not apply for $\mathrm{dG}$ velocity approximations. We have collected all these results in Table 1 , where the convergence rate of the different error quantities is indicated for all the methods introduced above, in terms of $k$ and $l$. We have also marked the results that are not always true, and in which cases these bounds are false.

All these rates of convergence allow us to draw some recommendations about the method to use, depending on the order of the velocity-pressure approximation, that is to say, the pair $(k, l)$ :

- $k<l$ : This situation has limited interest since it is not used in flow in porous media applications and because of the fact that the velocity field cannot be approximated by piecewise constant velocities in our analysis. In any case, Method A should be the one to take in this case. This method becomes optimal for $k=l-1$ with $l>1$ since $k>0$ has to be assumed. On the other hand, this is the natural method for the mixed Laplacian formulation.

- $k=l$ : For equal velocity-pressure approximations Method B is the most accurate one. Furthermore, it is optimal for conforming FE approximations. When using Method D, the choice of $k=l$ is the best one. Anyways, this method is far from being optimal and is always worse than Method A. The nice property of Method D is the fact that it exhibits the same stability as the continuous problem for $\boldsymbol{f} \in L^{2}(\Omega)^{d}$ (see Remark 2.2).

- $k>l$ : Method $\mathrm{C}$ is the one that performs best when using this fairly used choice. In fact, the method is optimal when $k=l+1$ for any interpolation pair. It is important to remark that Method $C$ is the only one that allows us to take $l=0$. Afar as we know, this is the first stabilized formulation of the Darcy problem that allows to use piecewise constant pressures. Furthermore, this method has been proved to be optimal for the Stokes-Darcy problem in Ref. [4]. ${ }^{5}$

\footnotetext{
${ }^{5}$ Obviously, the use of piecewise constant pressures for the finite element approximation of the Darcy problem is not new, since this is the choice for the pressure subspace when using Raviart-Thomas or Douglas-Brezzi-Marini inf-sup stable finite elements (see Refs. [5,25]). The novelty of our formulation is the fact that it allows arbitrary finite element spaces for the velocity (which is certainly not the case of inf-sup stable methods) and piecewise constant pressures.
} 
Table 1

Convergence rates according to the choice of the length scale in the stabilization parameters. When using piecewise constant pressures, the results marked with $(\ddagger)$ are only true for large enough $c_{u}$. The results marked with ( $\star$ ) are only true for $V_{h} \subset \mathcal{C}^{0}(\Omega)$. The results marked with $(\dagger)$ only apply to the ASGS formulation.

\begin{tabular}{|c|c|c|c|c|}
\hline $\begin{array}{l}\text { Method } \\
\left(/_{p}, /_{u}\right) \approx\end{array}$ & $\begin{array}{l}\text { A } \\
(h, h)\end{array}$ & $\begin{array}{l}\text { B } \\
\left(L_{0}^{1 / 2} h^{1 / 2}, L_{0}^{1 / 2} h^{1 / 2}\right)\end{array}$ & $\begin{array}{l}\mathrm{C} \\
\left(L_{0}, L_{0}\right)\end{array}$ & $\begin{array}{l}\mathrm{D} \\
\left(L_{0}, h\right)\end{array}$ \\
\hline$\left\|e_{u}\right\|$ & $h^{k+1}+h^{l}$ & $h^{k+1 / 2}+h^{l+1 / 2}$ & $h^{k}+h^{l+1}$ & $h^{k}+h^{l}(\dagger)$ \\
\hline$\left\|e_{u}\right\|$ (duality) & $h^{k+1}+h^{l}$ & $h^{k+1}+h^{l+1}(\star)$ & $h^{k}+h^{l+1}$ & $h^{k}+h^{l}(\dagger)$ \\
\hline$\left\|e_{p}\right\|$ & $h^{k+1}+h^{l}$ & $h^{k+1 / 2}+h^{l+1 / 2}$ & $h^{k}+h^{l+1}$ & $h^{k}+h^{l}(\dagger)$ \\
\hline$\left\|e_{p}\right\|$ (duality) & $h^{k+2}+h^{l+1}(\ddagger)$ & $h^{k+1}+h^{l+1}$ & $h^{k}+h^{l+1}$ & $h^{k}+h^{l}(\dagger)$ \\
\hline$\left\|\nabla \cdot e_{u}\right\|$ & $h^{k}+h^{l-1}$ & $h^{k}+h^{l}$ & $h^{k}+h^{l+1}$ & $h^{k}+h^{l}(\dagger)$ \\
\hline$\left\|\nabla e_{p}\right\|$ & $h^{k+1}+h^{l}$ & $h^{k}+h^{l}$ & $h^{k-1}+h^{l}$ & $h^{k}+h^{l}(\dagger)$ \\
\hline Optimal $(k, l)$ & $k+1=l$ & $k=l$ & $k=l+1$ & $k=l$ \\
\hline
\end{tabular}

Table 2

Experimental convergence rates for the ASGS method according to the choice of the length scale in the stabilization parameters. The $P 1^{c} / P 1^{d}$ pair.

\begin{tabular}{lllll}
\hline $\begin{array}{l}\text { Method } \\
\ell_{p}, \ell l_{u}=\end{array}$ & $\mathrm{A}$ & $\mathrm{B}$ & $\mathrm{C}$ & $\mathrm{D}$ \\
$\left\|e_{u}\right\|$ & $1.50(1)$ & $1.86(2)$ & $L_{0}, L_{0}$ & $L_{0}, h$ \\
$\left\|e_{p}\right\|$ & $2.05(2)$ & $2.39(2)$ & $1.89(1)$ & $1.69(1)$ \\
$\left\|\nabla \cdot e_{u}\right\|$ & $1.32(-)$ & $1.47(1)$ & $1.67(1)$ & $2.07(1)$ \\
$\left\|\nabla e_{p}\right\|$ & $1.04(1)$ & $0.99(1)$ & $1.53(1)$ & $1.76(1)$ \\
\hline
\end{tabular}

\section{Numerical testing}

In this section we carry out some numerical experiments in order to check the theoretical convergence rates proved in Sections 5 and 6. We have considered both the ASGS and the OSS techniques with all the possible choices of the stabilization parameters that have been analyzed previously. Let us denote the spaces of discontinuous piecewise linear functions as $P 1^{d}$, continuous piecewise linear functions as $P 1^{c}$ and piecewise constant (obviously discontinuous) functions as $P 0^{d}$. This notation is used for both the velocity and the pressure interpolation. With regard to the FE approximations, we have considered four velocity-pressure pairs: $P 1^{c} / P 0^{d}, P 1^{c} / P 1^{d}, P 1^{d} /$ $P 0^{d}$ and $P 1^{d} / P 1^{d}$. Numerical experiments for the $P 1^{c} / P 1^{c}$ pair have not been included for the sake of conciseness, but they can be found in Ref. [4] in the frame of the Stokes-Darcy system.

All test problems are defined in the domain $\Omega \equiv(0,1) \times(0,1)$. We have considered structured and regular meshes. The family of FE partitions used in the convergence analysis consists of 3200,7200 and 12,800 linear triangular elements.

The definition of the stabilization parameters in Eq. (19) include the algorithmic constants $c_{u}$ and $c_{p}$ and a characteristic length $L_{0}$. Let us consider $c_{u}=\gamma c_{p}$. We have used $c_{p}=2$ and $L_{0}=0.1 \sqrt[d]{\operatorname{meas}(\Omega)}$ in all cases. Based on numerical experimentation, we have taken $\gamma=1$ for methods $A$ and $B$ and $\gamma=0.1$ for methods $C$ and $D$.

In order to evaluate the error introduced by the numerical approximations, we have solved a test problem with analytical solution:

$u=(-2 \pi \cos (2 \pi x) \sin (2 \pi y),-2 \pi \sin (2 \pi x) \cos (2 \pi y))$,

$p=\sin (2 \pi x) \sin (2 \pi y)$

Table 3

Experimental convergence rates for the OSS method according to the choice of the length scale in the stabilization parameters. The $P 1^{c} / P 1^{d}$ pair.

\begin{tabular}{llll}
\hline Method & $\mathrm{A}$ & $\mathrm{B}$ & $\mathrm{C}$ \\
$\ell_{p}, \ell_{u}=$ & $h, h$ & $L_{0}^{1 / 2} h^{1 / 2}, L_{0}^{1 / 2} h^{1 / 2}$ & $L_{0}, L_{0}$ \\
\hline$\left\|e_{u}\right\|$ & $1.78(1)$ & $1.91(2)$ & $1.77(1)$ \\
$\left\|e_{p}\right\|$ & $1.96(2)$ & $2.34(2)$ & $1.69(1)$ \\
$\left\|\nabla \cdot e_{u}\right\|$ & $0.65(-)$ & $1.44(1)$ & $1.51(1)$ \\
$\left\|\nabla e_{p}\right\|$ & $1.12(1)$ & $0.99(1)$ & $0.03(-)$ \\
\hline
\end{tabular}

Table 4

Experimental convergence rates for the ASGS method according to the choice of the length scale in the stabilization parameters. The $P 1^{d} / P 1^{d}$ pair.

\begin{tabular}{lllll}
\hline $\begin{array}{l}\text { Method } \\
\ell_{p}, \ell_{u}=\end{array}$ & $\mathrm{A}$ & $\mathrm{B}$ & $\mathrm{C}$ & $\mathrm{D}$ \\
\hline$\left\|e_{u}\right\|$ & $1.00(1)$ & $1.94(1.5)$ & $L_{0}, L_{0}$ & $L_{0}, h$ \\
$\left\|e_{p}\right\|$ & $1.99(2)$ & $2.31(2)$ & $1.98(1)$ & $1.00(1)$ \\
$\left\|\nabla \cdot e_{u}\right\|$ & $0.58(-)$ & $1.01(1)$ & $1.59(1)$ & $1.98(1)$ \\
$\left\|\nabla e_{p}\right\|$ & $1.05(1)$ & $0.98(1)$ & $1.21(1)$ & $1.04(1)$ \\
\hline
\end{tabular}

Table 5

Experimental convergence rates for the OSS method according to the choice of the length scale in the stabilization parameters. The $P 1^{d} / P 1^{d}$ pair.

\begin{tabular}{llll}
\hline Method & $\mathrm{A}$ & $\mathrm{B}$ & $\mathrm{C}$ \\
$\ell_{p}, \ell_{u}=$ & $h, h$ & $L_{0}^{1 / 2} h^{1 / 2}, L_{0}^{1 / 2} h^{1 / 2}$ & $L_{0}, L_{0}$ \\
\hline$\left\|e_{u}\right\|$ & $1.79(1)$ & $2.00(2)$ & $1.99(1)$ \\
$\left\|e_{p}\right\|$ & $2.19(2)$ & $2.33(2)$ & $1.47(1)$ \\
$\left\|\nabla \cdot e_{u}\right\|$ & $0.09(-)$ & $1.07(1)$ & $1.06(1)$ \\
$\left\|\nabla e_{p}\right\|$ & $1.62(1)$ & $1.02(1)$ & $0.03(-)$ \\
\hline
\end{tabular}

that can be obtained with the appropriate choice of $\boldsymbol{f}, g$ and boundary conditions. This test has been used in Ref. [23]. The analytical solution is obtained for $\boldsymbol{f}=\mathbf{0}$. Let us remark that, due to the regularity of the solution, only the normal component of the velocity can be enforced on the boundary.

With all the experimental convergence rates obtained, we want to support the recommendations of the previous sections:

- $k<l$ : The lower order pair that could be used is the $P 1^{d} / P 2^{d}$ (or its continuous counterpart); since this FE space is of limited interest, we do not consider this case in the numerical experiments.

- $k=l$ : The numerical orders of convergence obtained for the $P 1^{c} / P 1^{d}$ case are collected in Table 2 for the ASGS method and in Table 3 for the OSS method. The theoretical order of convergence is indicated in parentheses and $(-)$ is used when no convergence is expected. It becomes clear from these results that Method B is the optimal one. Anyway, all the methods exhibit super-convergence. The results for the $P 1^{d} / P 1^{d}$ case are shown in Tables 4 and 5 for the ASGS and the OSS methods, respectively. Again, the superiority of Method B is clear; Method C still keeps super-convergence. Methods A and D have lost this super-convergence for the ASGS formulation but Method A keeps it for the OSS approach.

- $k=l-1$ : The results for the $P 1^{c} / P 0^{d}$ interpolation are included in Table 6 for the ASGS method and in Table 7 for the OSS formulation. As expected, when using piecewise constant pressures, Methods A and D fail to converge. The superiority of Method $C$ is even clearer than expected thanks to super-convergence. Method B only converges in $L^{2}$-norms, and always exhibits lower orders of convergence than Method C. For $P 1^{d} / P 0^{d}$, with discontinuous velocities, the orders of convergence can be found in Table 8 for ASGS method and Table 9 for the OSS approach. Again, Method C is clearly the method to use.

Table 6

Experimental convergence rates for the ASGS method according to the choice of the length scale in the stabilization parameters. The $P 1^{c} / P 0^{d}$ pair.

\begin{tabular}{lllll}
\hline $\begin{array}{l}\text { Method } \\
\ell_{p}, \ell_{u}=\end{array}$ & $\mathrm{A}$ & $\mathrm{B}$ & $\mathrm{C}$ & $\mathrm{D}$ \\
$\left\|e_{u}\right\|$ & $-0.09(-)$ & $0.74(1)$ & $1.84(1)$ & $-0.03(-)$ \\
$\left\|e_{p}\right\|$ & $0.01(1)$ & $0.94(1)$ & $1.88(1)$ & $-0.01(-)$ \\
$\left\|\nabla \cdot e_{u}\right\|$ & $-0.38(-)$ & $0.48(-)$ & $1.54(1)$ & $-0.03(-)$ \\
$\left\|\nabla e_{p}\right\|$ & $-0.98(-)$ & $-0.03(-)$ & $0.54(-)$ & $-0.99(-)$ \\
\hline
\end{tabular}


Table 7

Experimental convergence rates for the OSS method according to the choice of the length scale in the stabilization parameters. The $P 1^{c} / P 0^{d}$ pair.

\begin{tabular}{llll}
\hline Method & $\mathrm{A}$ & $\mathrm{B}$ & $\mathrm{C}$ \\
$\ell_{p}, \ell_{u}=$ & $h, h$ & $L_{0}^{1 / 2} h^{1 / 2}, L_{0}^{1 / 2} h^{1 / 2}$ & $L_{0}, L_{0}$ \\
\hline$\left\|e_{u}\right\|$ & $-0.09(-)$ & $0.75(1)$ & $1.84(1)$ \\
$\left\|e_{p}\right\|$ & $0.01(1)$ & $0.95(1)$ & $1.89(1)$ \\
$\left\|\nabla \cdot e_{u}\right\|$ & $-0.38(-)$ & $0.49(-)$ & $1.54(1)$ \\
$\left\|\nabla e_{p}\right\|$ & $-0.98(-)$ & $-0.03(-)$ & $0.54(-)$ \\
\hline
\end{tabular}

Table 8

Experimental convergence rates for the ASGS method according to the choice of the length scale in the stabilization parameters. The $P 1^{d} / P 0^{d}$ pair.

\begin{tabular}{lllll}
\hline Method & $\mathrm{A}$ & $\mathrm{B}$ & $\mathrm{C}$ & $\mathrm{D}$ \\
$\ell_{p}, \ell_{u}=$ & $h, h$ & $L_{0}^{1 / 2} h^{1 / 2}, L_{0}^{1 / 2} h^{1 / 2}$ & \multicolumn{1}{l}{$L_{0}, L_{0}$} & \multicolumn{1}{l}{$L_{0}, h$} \\
\hline$\left\|e_{u}\right\|$ & $-0.03(-)$ & $0.80(0.5)$ & $1.86(1)$ & $0.07(-)$ \\
$\left\|e_{p}\right\|$ & $-0.02(1)$ & $0.84(1)$ & $1.83(1)$ & $0.01(-)$ \\
$\left\|\nabla \cdot e_{u}\right\|$ & $-0.37(-)$ & $0.48(-)$ & $1.06(1)$ & $-0.12(-)$ \\
$\left\|\nabla e_{p}\right\|$ & $-0.99(-)$ & $-0.14(-)$ & $0.83(-)$ & $-0.98(-)$ \\
\hline
\end{tabular}

These results are a numerical evidence of the recommendations stated in the previous section.

\section{Conclusions}

In this article we have motivated a set of stabilized methods for the numerical approximation of the Darcy problem in mixed form for arbitrary continuous and discontinuous finite element approximations of velocities and pressures. A continuous range of finite element methods have been proposed, depending on the choice of a length scale. The stability and convergence analyses have been performed in a general setting that include all the stabilized methods that have been designed. We have also used duality arguments to obtain improved error estimates in $L^{2}$-norms. The theoretical analysis has allowed us to draw recommendations about the method to be used, depending on the order of approximation of velocities and pressures. In particular, four cases have been implemented and these recommendations have been proved to be accurate using numerical experimentation.

Method A mimics the mixed primal formulation and has been proposed in Refs. $[20,23,24]$. This method has been proved to be optimal for FE pressure approximations of order higher than the one used for the velocity, which is not a common choice in practice. In this frame, we have motivated two new methods that are particularly interesting in flow in porous media applications. Method B mimics an intermediate setting between the primal and dual formulation, but turns out to be the optimal choice for equal-order velocity-pressure approximations. Method $C$ is particularly well suited when the order of the velocity FE space is one order higher than the pressure one. As far as we know, this is the first stabilized method that allows piecewise constant pressures, and the only one that mimics the dual primal formulation of the Darcy problem, which is the one in which Raviart-Thomas or Douglas-Brezzi-Marini inf-sup stable finite ele-

\section{Table 9}

Experimental convergence rates for the OSS method according to the choice of the length scale in the stabilization parameters. The $P 1^{d} / P 0^{d}$ pair.

\begin{tabular}{llll}
\hline $\begin{array}{l}\text { Method } \\
\ell_{p}, \ell_{u}=\end{array}$ & $\mathrm{A}$ & $\mathrm{B}$ & $\mathrm{C}$ \\
\hline$\left\|e_{u}\right\|$ & $h, h$ & $L_{0}^{1 / 2} h^{1 / 2}, L_{0}^{1 / 2} h^{1 / 2}$ & $L_{0}, L_{0}$ \\
$\left\|e_{p}\right\|$ & $-0.02(-)$ & $0.87(1)$ & $1.89(1)$ \\
$\left\|\nabla \cdot e_{u}\right\|$ & $-0.04(1)$ & $0.78(1)$ & $1.85(1)$ \\
$\left\|\nabla e_{p}\right\|$ & $-0.90(-)$ & $-0.01(-)$ & $0.91(1)$ \\
\hline
\end{tabular}

ments work. Furthermore, these methods do not require additional regularity assumptions over the data, a difference with respect to the stabilized formulation in Ref. [15], that required $\sigma \in \mathcal{C}^{1}(\Omega)$, which is false in real applications in flow in porous medium.

Compared to inf-sup stable finite element methods, our formulation allows arbitrary FE spaces for velocities and pressures. With our formulation, a stabilized FE solver for the Navier-Stokes equations leads to a Darcy solver by the simple modification of the subroutine that evaluates the stabilization parameters. This makes this approach very interesting in terms of implementation time, and it is particularly well-suited for coupled problems that involve Navier-Stokes and Darcy problems.

Future work involves the application of our formulation to realistic engineering applications and the extension to the Biot system for the numerical approximation of poroelastic materials.

\section{Acknowledgment}

The first author's research was supported by the European Community through the Marie Curie contract NanoSim (MOIF-CT2006-039522).

\section{References}

[1] T. Arbogast, D.S. Brunson, A computatonal method for approximating a DarcyStokes system governing a vuggy porous medium, Computational Geosciences 11 (2007) 207-218

[2] D.N. Arnold, D. Boffi, R.S. Falk, Quadrilateral $H$ (div) finite elements, SIAM Journal on Numerical Analysis 42 (2005) 2429-2451.

[3] S. Badia, R. Codina, On a multiscale approach to the transient Stokes problem: dynamic subscales and anisotropic space-time discretization, Applied Mathematics and Computation 207 (2009) 415-433.

[4] S. Badia, R. Codina, Unified stabilized finite element formulations for the Stokes and the Darcy problems, SIAM Journal on Numerical Analysis 47 (3) (2009) 1977-2000.

[5] F. Brezzi, M. Fortin, Mixed and Hybrid Finite Element Methods, Springer Verlag, 1991.

[6] F. Brezzi, T.J.R. Hughes, L.D. Marini, A. Masud, Mixed discontinuous Galerkin methods for Darcy flow, Journal of Scientific Computing 22-23 (2005) 119-145.

[7] P.G. Ciarlet, The Finite Element Method for Elliptic Problems, North-Holland, Amsterdam, 1978.

[8] B. Cockburn, J. Guzmán, H. Wang, Superconvergent discontinuous Galerkin methods for second-order elliptic problems, Mathematics of Computation 78 (265) (2009) 1-24.

[9] R. Codina, Stabilization of incompressibility and convection through orthogonal sub-scales in finite element methods, Computer Methods in Applied Mechanics and Engineering 190 (2000) 1579-1599.

[10] R. Codina, Stabilized finite element approximation of transient incompressible flows using orthogonal subscales, Computer Methods in Applied Mechanics and Engineering 191 (2002) 4295-4321.

[11] R. Codina, Analysis of a stabilized finite element approximation of the Oseen equations using orthogonal subscales, Applied Numerical Mathematics 58 (2008) 264-283.

[12] R. Codina, Finite element approximation of the hyperbolic wave equation in mixed form, Computer Methods in Applied Mechanics and Engineering 197 (1316) (2008) 1305-1322.

[13] R. Codina, J. Blasco, Analysis of a pressure-stabilized finite element approximation of the stationary Navier-Stokes equations, Numerische Mathematik 87 (2000) 59-81.

[14] R. Codina, J. Principe, J. Baiges, Subscales on the element boundaries in the variational two-scale finite element method, Computer Methods in Applied Mechanics and Engineering 198 (5-8) (2009) 838-852.

[15] M.R. Correa, A.F.D. Loula, Stabilized velocity post-processings for Darcy flow in heterogeneous porous media, Communications in Numerical Methods in Engineering 23 (2007) 461-489.

[16] M.R. Correa, A.F.D. Loula, Unconditionally stable mixed finite element methods for Darcy flow, Computer Methods in Applied Mechanics and Engineering 197 (2008) 1525-1540.

[17] A.L.G.A. Coutinho, C.M. Dias, J.L.D. Alves, L. Landau, A.F.D. Loula, S.M.C. Malta, R.G.S Castro, E.L.M. Garcia, Stabilized methods and post-processing techniques for miscible displacements, Computer Methods in Applied Mechanics and Engineering 193 (2004) 1421-1436.

[18] A. Ern, J.L. Guermond, Theory and Practice of Finite Elements, Springer-Verlag, 2004.

[19] T.J.R. Hughes, Multiscale phenomena: Green's function, the Dirichlet-to-Neumann formulation, subgrid scale models, bubbles and the origins of stabilized formulations, Computer Methods in Applied Mechanics and Engineering 127 (1995) 387-401. 
[20] T.J.R. Hughes, A. Masud, J. Wan, A stabilized mixed discontinuous Galerkin method for Darcy flow, Computer Methods in Applied Mechanics and Engineering 195 (2006) 3347-3381.

[21] T.J.R. Hughes, G. Sangalli, Variational multiscale analysis: the fine-scale Green's function, projection, optimization, localization, and stabilized methods, SIAM Journal on Numerical Analysis 45 (2) (2007) 539-557.

[22] K.A. Mardal, X.C. Tai, R. Winther, A robust finite element method for Darcy-Stokes flow, SIAM Journal on Numerical Analysis 40 (2002) 1605-1631.

[23] A. Masud, T.J.R. Hughes, A stabilized mixed finite element method for Darcy flow, Computer Methods in Applied Mechanics and Engineering 191 (2002) 4341-4370.
[24] K.B. Nakshatrala, D.Z. Turner, K.D. Hjelmstad, A. Masud, A stabilized mixed finite element method for Darcy flow based on a multiscale decomposition of the solution, Computer Methods in Applied Mechanics and Engineering 195 (33-36) (2006) 4036-4049.

[25] P.A. Raviart, J.M. Thomas, A mixed-finite element method for second order elliptic problems, volume Mathematical aspects of the finite element method, Lecture Notes in Mathematics, Springer, New York, 1977.

[26] R. Stenberg, On some techniques for approximating boundary conditions in the finite element method, Journal of Computational and Applied Mathematics 63 (1995) 139-148. 